\title{
Estimation of errors in the inverse modeling of accidental release of atmospheric pollutant: Application to the reconstruction of the cesium-137 and iodine-131 source terms from the Fukushima Daiichi power plant
}

\author{
Victor Winiarek, ${ }^{1,2}$ Marc Bocquet, ${ }^{1,2}$ Olivier Saunier, ${ }^{3}$ and Anne Mathieu ${ }^{3}$ \\ Received 27 September 2011; revised 19 January 2012; accepted 23 January 2012; published 9 March 2012.
}

[1] A major difficulty when inverting the source term of an atmospheric tracer dispersion problem is the estimation of the prior errors: those of the atmospheric transport model, those ascribed to the representativity of the measurements, those that are instrumental, and those attached to the prior knowledge on the variables one seeks to retrieve. In the case of an accidental release of pollutant, the reconstructed source is sensitive to these assumptions. This sensitivity makes the quality of the retrieval dependent on the methods used to model and estimate the prior errors of the inverse modeling scheme. We propose to use an estimation method for the errors' amplitude based on the maximum likelihood principle. Under semi-Gaussian assumptions, it takes into account, without approximation, the positivity assumption on the source. We apply the method to the estimation of the Fukushima Daiichi source term using activity concentrations in the air. The results are compared to an L-curve estimation technique and to Desroziers's scheme. The total reconstructed activities significantly depend on the chosen method. Because of the poor observability of the Fukushima Daiichi emissions, these methods provide lower bounds for cesium-137 and iodine-131 reconstructed activities. These lower bound estimates, $1.2 \times 10^{16} \mathrm{~Bq}$ for cesium-137, with an estimated standard deviation range of $15 \%-20 \%$, and $1.9-3.8 \times 10^{17} \mathrm{~Bq}$ for iodine-131, with an estimated standard deviation range of $5 \%-10 \%$, are of the same order of magnitude as those provided by the Japanese

Nuclear and Industrial Safety Agency and about 5 to 10 times less than the Chernobyl atmospheric releases.

Citation: Winiarek, V., M. Bocquet, O. Saunier, and A. Mathieu (2012), Estimation of errors in the inverse modeling of accidental release of atmospheric pollutant: Application to the reconstruction of the cesium-137 and iodine-131 source terms from the Fukushima Daiichi power plant, J. Geophys. Res., 117, D05122, doi:10.1029/2011JD016932.

\section{Introduction}

\subsection{Estimation of Errors in the Inverse Modeling of Accidental Release}

[2] Like any inverse modeling problem, the reconstruction of the source term of an accidental release of atmospheric tracer depends on the balance between the information load in the observation set, and the number of source parameters to retrieve, as well as the nature of the source-receptor relationship between them, provided by a numerical model of atmospheric transport (ATM). The source-receptor relationship reads

$$
\boldsymbol{\mu}=\mathbf{H} \boldsymbol{\sigma}+\boldsymbol{\epsilon}
$$

\footnotetext{
${ }^{1}$ CEREA, École des Ponts ParisTech-EDF R\&D, Université Paris-Est, Marne la Vallée, France.

${ }^{2}$ INRIA Rocquencourt Research Centre, Paris, France.

${ }^{3}$ Institute of Radiation Protection and Nuclear Safety, Fontenay-auxRoses, France.

Copyright 2012 by the American Geophysical Union. 0148-0227/12/2011JD016932
}

where $\boldsymbol{\mu}$ in $\mathbb{R}^{d}$ is the measurement vector, $\boldsymbol{\sigma}$ in $\mathbb{R}^{N}$ is the source vector, and $\mathbf{H}$ is the Jacobian matrix of the transport model which is linear in this context for gaseous and particulate matter. It is clear that $\mathbf{H}$ also incorporates the observation operator. The vector $\boldsymbol{\epsilon}$ in $\mathbb{R}^{d}$, called the observation error in this article, represents the instrumental errors, as well as the representativeness errors. By equation (1), it accounts for model errors as well. For the transport of tracer at regional to global scale, and in particular long-range radionuclides, it is well known that the Jacobian matrix is ill conditioned because of the dispersive nature of large-scale transport [Enting, 2002]. As a result, the source-receptor relationship equation (1) constitutes an ill-posed inverse problem.

[3] Additionally, in an accidental context, when remote sensing means are essentially unavailable such as in the case of radionuclides, the number of observations is limited.

[4] One solution to alleviate the lack of constraints is to parameterize the source with a very limited number of variables. This corresponds to the so-called parametric methods. De facto, this regularizes the inverse problem, and even allows to compute posterior probability density function for 
the parameters through stochastic sampling techniques [Delle Monache et al., 2008; Yee et al., 2008]. However, it is highly constraining: if the true source does not abide to this parametric model, the inverse may fail, have multiple minima or be meaningless.

[5] Another route which has been followed for the retrieval of tracer sources, is of nonparametric nature: one seeks to retrieve a source/emission field. It must ultimately be discretized, which leads to a set of variables but this set can be large, and even significantly larger than the number of observations.

[6] The latter approach can rely on the set of techniques developed by the field of geophysical data assimilation. It is very flexible since the source is not parameterized a priori but it has its own constraints. First, under simple Gaussian statistical assumptions on the observation errors, it essentially relies in the minimization of

$$
\mathcal{L}(\boldsymbol{\sigma})=\frac{1}{2}(\boldsymbol{\mu}-\mathbf{H} \boldsymbol{\sigma})^{\mathrm{T}} \mathbf{R}^{-1}(\boldsymbol{\mu}-\mathbf{H} \boldsymbol{\sigma}),
$$

where $\mathbf{R}$ is the observation error covariance matrix: $\mathbf{R}=$ $\mathrm{E}\left[\boldsymbol{\epsilon} \boldsymbol{\epsilon}^{\mathrm{T}}\right]$, where $\boldsymbol{\epsilon}$ has been defined by equation (1). One simple choice it to set $\mathbf{R}$ to $r^{2} \mathbf{I}_{d}$, assuming no correlation in the observation errors. With a large number of source parameters, and/or an ill-conditioned physical model representation $(\mathbf{H})$, the minimization of equation (2) offers infinitely many solutions. To regularize the inverse problem, one usually adds a Tikhonov term to the cost function

$$
\mathcal{L}(\boldsymbol{\sigma})=\frac{1}{2}(\boldsymbol{\mu}-\mathbf{H} \boldsymbol{\sigma})^{\mathrm{T}} \mathbf{R}^{-1}(\boldsymbol{\mu}-\mathbf{H} \boldsymbol{\sigma})+\frac{1}{2}\left(\boldsymbol{\sigma}-\boldsymbol{\sigma}_{b}\right)^{\mathrm{T}} \mathbf{B}^{-1}\left(\boldsymbol{\sigma}-\boldsymbol{\sigma}_{b}\right),
$$

which formally guarantees the existence of a unique solution, at the cost of introducing additional parameters: $\mathbf{B}$ is the background error covariance matrix, and $\boldsymbol{\sigma}_{b}$ is the first guess for the source (known a priori).

[7] Unfortunately, as opposed to the forecasting context of data assimilation in meteorology, the background information specified by $\boldsymbol{\sigma}_{b}$ and $\mathbf{B}$ is uncertain. In atmospheric chemistry data assimilation that focuses on the emission retrieval, the first guess $\sigma_{b}$ is usually provided by an inventory built up from survey (anthropogenic sources), or from a vegetation model (biogenic sinks and sources). As a result, $\mathbf{B}$ can be roughly estimated from these bottom-up methodologies. Correlations in the prior source errors (related to the off-diagonal entries of $\mathbf{B}$ ) are usually used to constrain the inverse modeling problem which is a smooth way of aggregating variables. More fundamentally they may correspond to correlations in the errors when building the inventory. Recent examples using these concepts are given by Villani et al. [2010, and references therein] and Wu et al. [2011, and references therein] for greenhouses gas flux inversions and by Elbern et al. [2007, and references therein] for air quality emission inverse modeling.

[8] In the context of an accidental release, the status of $\sigma_{b}$ and $\mathbf{B}$ significantly differ, because it is difficult to form a precise idea on the source a priori. The first guess $\sigma_{b}$ is often set to zero. One reason is that most of the emission rates are likely to be zero. Other reasons are discussed by Bocquet
[2005] and Davoine and Bocquet [2007]. Alternatively one can contemplate using a first guess built from a model of the accident. However, an estimation of the errors made by this model needed to define $\mathbf{B}$ is even more uncertain than the models used to build up inventories of nonaccidental emission fields. Besides, one must be aware that such a prior $\sigma_{b}$ is usually calibrated by observations which could lead to a multiple and uncontrolled use of observations (often referred to as an inversion crime in inverse modeling). In addition, the use of $\sigma_{b}$ built from a model of the accident will lead to a nonindependent estimation of the source term. That is why we favor the choice $\sigma_{b}=\mathbf{0}$ in this article.

[9] Still in the context of an accidental release, the presence of off-diagonal terms in $\mathbf{B}$, which corresponds to correlation in the estimated errors, would imply some persistence in the emission rates and the way the model makes errors about them. For accidental release this persistence is often negligible. For instance in the case of the Fukushima Daiichi accident, the gamma dose measurements at the nuclear power plant site reveals that the source term must certainly be composed of uncorrelated events.

[10] That is why we opt for the simple uncorrelated statistical model: $\mathbf{B}=m^{2} \mathbf{I}_{N}$. However, the choice of scalar $m$ remains crucial. In the case of Chernobyl source term reconstruction, Davoine and Bocquet [2007] have shown that the total reconstructed activity is highly dependent on the choice of $r$ and $m$. This required the use of a hyperparameter $(r$ and $m)$ estimation technique. Davoine and Bocquet [2007], Krysta et al. [2008] and Saide et al. [2011] used the L-curve technique of Hansen [1992] to either estimate the ratio $r / m$ or both parameters. In the context of the Chernobyl inverse modeling, Bocquet [2012] has shown that even with a number of source parameters three times inferior to the number of observations, the poor observability of some of the source variables still leads to an ill-conditioned problem with a too high sensitivity of the results to the hyperparameters, so that a hyperparameter estimation technique is required.

[11] Recently, methodological developments of data assimilation in meteorology have focused on the estimation of such hyperparameters. They are based on either a maximum likelihood estimation technique or a cross-validation technique: Mitchell and Houtekamer [1999], Chapnik et al. [2004, 2006], Anderson [2007], and Li et al. [2009], to cite just a few. These techniques allow online estimation of the errors. They can lead to significant improvement of the forecasting skills. These techniques have also made their way to atmospheric chemistry inverse modeling, using for instance the $\chi^{2}$ criterion [Ménard et al., 2000; Elbern et al., 2007] or the maximum likelihood principle [Michalak et al., 2004].

[12] Our inverse modeling point of view, where the background/regularization is poorly known, is different but the mathematical approach remains relevant to our problem. In this article, we would like to adapt these hyperparameter estimation techniques to the demanding case of the reconstruction of the Fukushima Daiichi source term.

\subsection{Fukushima Daiichi Accident}

[13] On 11 March, 05:46 UTC, a magnitude $9.0\left(\mathrm{M}_{\mathrm{w}}\right)$ undersea megathrust earthquake occurred about $70 \mathrm{~km}$ east 
of the Oshika Peninsula (Japan) in the Pacific Ocean. About an hour later, an extremely destructive tsunami hit the Pacific coastline of Japan's northern islands and resulted in the loss of thousands of lives and devastated entire towns.

[14] These two combined disasters caused the automatic shutdown of eleven nuclear reactors in Japan, located on four nuclear power plants (Fukushima Daiichi, Fukushima Daini, Onagawa and Tokai power plants). The reactor cooling process was then operated through diesel backup power systems. Nevertheless, the damages caused by the earthquake and the tsunami rendered the backup cooling systems inoperative in the Fukushima Daiichi power plant and the reactors began to overheat.

[15] The Fukushima Daiichi power plant has six nuclear reactors. At the time of the earthquake, reactor 4 had been defueled and reactors 5 and 6 were in a cold shut-down for planned maintenance.

[16] In the hours and days that followed, reactors 1, 2 and 3 experienced at least partial meltdown and hydrogen explosions that destroyed the upper cladding of the buildings housing reactors. Multiple fires broke out at reactor 4 . Additionally fuel rods stored in pools in each reactor building began to overheat as water levels in the pools dropped.

[17] All these events caused a massive discharge of radioactive materials in the atmosphere, especially during the first 2 weeks, which has to be quantified in order to estimate the sanitary and environmental impact of the accident.

\subsection{Objectives and Outline}

[18] Our objective is twofold. The final objective is to estimate the Fukushima Daiichi radionuclide source term available for long-range transport with a health impact, using radionuclides activity concentrations in the air. Because of the small number of publicly released observations, we need to extend the current available methodologies to estimate the errors in order to take into account all the information available, such as the positivity of the source term. This is the intermediary objective.

[19] In section 2, we briefly recall what the current available methodologies for the inverse modeling of accidental release of pollutant are. The amplitude of the statistics of the errors that is required by these algorithms was shown to be crucial for a proper estimation. Hence, the techniques to estimate these errors, are briefly recalled: L-curve, generalized cross-validation, and maximum likelihood. We propose a generalization of the maximum likelihood scheme, that rigorously takes into account the positivity of the source. How to estimate the posterior errors, attached to the estimated sources, is also explained.

[20] In section 3, we apply the new method, as well as former ones, to the estimation of the atmospheric release of cesium-137 and iodine-131 from the Fukushima Daiichi plant. We demonstrate the poor observability of the release by the activity measurements, and provide lower bounds for the source term. From the methodological standpoint, the former schemes are shown to lead to significantly different estimates, and several pitfalls. The posterior uncertainty of the retrieved source is computed. Several sensitivity tests are performed. In particular we discuss the interest and impact of using a nonzero first guess.
[21] Results are summarized and conclusions are given in section 4 .

\section{Methodology: Modeling Errors}

\subsection{Observation Errors}

[22] Throughout this article, the observation errors, as defined by equation (1), which represent model, representativeness and instrumental errors altogether, are assumed Gaussian, with probability density function (pdf):

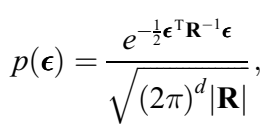

where $|\mathbf{R}|$ is the determinant of the error covariance matrix R. Quite naturally for errors of activity concentrations at different site, $\mathbf{R}$ is assumed diagonal. (However, in theory, one should include small correlations induced by model error.) Besides, as mentioned in the introduction, it is assumed they have the same variance, so that $\mathbf{R}=r^{2} \mathbf{I}_{d}$ (a property called homoscedasticity). More sophisticated approaches are possible [Abida and Bocquet, 2009; Winiarek et al., 2011]. However, the latter necessarily rely on more sophisticated models for the statistics of the observation errors. With a small set of observations, they could prove less robust and too contrived.

\subsection{Gaussian Modeling of Background Errors}

[23] As mentioned in the introduction we assume $\boldsymbol{\sigma}_{b}=\mathbf{0}$. Yet, for the sake of generality, we shall assume in this section that $\boldsymbol{\sigma}_{b}$ can be different. As a first step, we shall assume that the background errors are Gaussian:

$$
p(\boldsymbol{\sigma})=\frac{e^{-\frac{1}{2}\left(\boldsymbol{\sigma}-\boldsymbol{\sigma}_{b}\right)^{\mathrm{T}} \mathbf{B}^{-1}\left(\boldsymbol{\sigma}-\boldsymbol{\sigma}_{b}\right)}}{\sqrt{(2 \pi)^{N}|\mathbf{B}|}} .
$$

Because the background errors on the source are assumed uncorrelated, as discussed in the introduction, $\mathbf{B}$ is supposed to be diagonal. For the sake of simplicity, we shall assume these errors are homoscedastic: $\mathbf{B}=m^{2} \mathbf{I}_{N}$.

[24] Bayes's rule helps to formulate the inference, after the acquisition of the measurement vector $\boldsymbol{\mu}$ :

$$
\begin{aligned}
p(\boldsymbol{\sigma} \mid \boldsymbol{\mu})= & \frac{p(\boldsymbol{\mu} \mid \boldsymbol{\sigma}) p(\boldsymbol{\sigma})}{p(\boldsymbol{\mu})} \\
\propto & \exp \left\{-\frac{1}{2}(\boldsymbol{\mu}-\mathbf{H} \boldsymbol{\sigma})^{\mathrm{T}} \mathbf{R}^{-1}(\boldsymbol{\mu}-\mathbf{H} \boldsymbol{\sigma})\right. \\
& \left.-\frac{1}{2}\left(\boldsymbol{\sigma}-\boldsymbol{\sigma}_{b}\right)^{\mathrm{T}} \mathbf{B}^{-1}\left(\boldsymbol{\sigma}-\boldsymbol{\sigma}_{b}\right)\right\}
\end{aligned}
$$

To obtain the maximum a posteriori estimate, one should maximize $\ln p(\boldsymbol{\sigma} \mid \boldsymbol{\mu})$, which is equivalent to minimizing cost function equation (3). In this case, the result, known as the Best Linear Unbiased Estimator, is

$$
\boldsymbol{\sigma}_{a}=\boldsymbol{\sigma}_{b}+\mathbf{B} \mathbf{H}^{\mathrm{T}}\left(\mathbf{R}+\mathbf{H B H}^{\mathrm{T}}\right)^{-1}\left(\boldsymbol{\mu}-\mathbf{H} \boldsymbol{\sigma}_{b}\right) .
$$


The analysis of the posterior error leads to the analysis error covariance matrix:

$$
\mathbf{P}_{a}=\mathbf{B}-\mathbf{B H}^{\mathrm{T}}\left(\mathbf{R}+\mathbf{H B H}^{\mathrm{T}}\right)^{-1} \mathbf{H B} .
$$

\subsection{Semi-Gaussian Modeling of Background Errors}

[25] Enforcing the positivity of the source in inverse modeling is known to provide valuable information to the data assimilation system (see Bocquet et al. [2010] for a review). To do so, we can assume that the background errors are semi-Gaussian. The pdf of this normalized truncated normal distribution reads

$$
\begin{cases}\text { if } \quad \boldsymbol{\sigma} \geq 0 & p(\boldsymbol{\sigma})=\frac{e^{-\frac{1}{2}\left(\boldsymbol{\sigma}-\boldsymbol{\sigma}_{b}\right)^{\mathrm{T}} \mathbf{B}^{-1}\left(\boldsymbol{\sigma}-\boldsymbol{\sigma}_{b}\right)}}{\sqrt{(\pi / 2)^{N}|\mathbf{B}|}} \\ \text { otherwise } & p(\boldsymbol{\sigma})=0 .\end{cases}
$$

Using Bayes's rule equation (5) we observe that the inference is unchanged: one still has to minimize cost function equation (3). However, the positivity of $\sigma$ should be enforced during the numerical minimization, and there is no analytical solution to this problem.

[26] As a consequence, equation (8) is only an approximation to the posterior errors statistics, which are non-Gaussian. In this non-Gaussian statistical context, a proper way to sample the posterior error pdf is to use a Monte Carlo second-order sensitivity analysis. To compute sample $k$, one perturbs the observations $(\boldsymbol{\mu})$ and the first guess $\left(\boldsymbol{\sigma}_{b}=\mathbf{0}\right)$, with noises that respectively follow the observational and background error prior statistics, which leads to an analysis $\boldsymbol{\sigma}_{a}^{(k)}$ for the sample. Statistics can then be inferred from the empirical statistics of numerous draws. This will be used later.

\subsection{Estimation of the Hyperparameters}

[27] In the Gaussian case and with the particular forms given to $\mathbf{R}$ and $\mathbf{B}$, it should be noted that the analysis increment $\boldsymbol{\sigma}_{a}-\boldsymbol{\sigma}_{b}$ only depends on the ratio $r / m$, while $\mathbf{P}_{a}$ depends on both. A prior estimation of $r$ is possible but certainly very difficult since, in particular, it requires a fine knowledge of model errors. A prior estimation of $m$ is almost impossible, since it would require a large database of similar accidents, which fortunately does not exist. In the semiGaussian case, the analysis state and the analysis error covariance matrix depend on both $r$ and $m$.

[28] Hence, to estimate $r / m$, or both $r$ and $m$, one should rely on a posteriori techniques. Several techniques are available and are well described in recent books [Vogel, 2002; Hansen, 2010]. For instance, the general cross-validation method [Wahba, 1990, and references therein] is based on the idea that, with a proper estimation of the hyperparameters using a subset of the observations, the rest of the observations should be consistently accounted for. Another available method is the L-curve method, which is a semiempirical technique that determines the right balance between overfitting to the data, and oversmoothing by the regularization [Hansen, 1992]. The turning point is indicated by the corner formed by the plot of $\ln \left\|\boldsymbol{\mu}-\mathbf{H} \boldsymbol{\sigma}_{a}\right\|$ against $\ln \left\|\sigma_{a}-\sigma_{b}\right\|$. It has been used in the context of accidental atmospheric release inverse modeling by Davoine and Bocquet [2007], Krysta et al. [2008], Saide et al. [2011], and Bocquet [2012]. In the ETEX-I and Chernobyl cases, it was shown that only the use of such techniques was able to deliver an estimate of the source terms consistent with the officially reported emissions.

[29] Another approach is based on the maximum likelihood. As mentioned in the introduction, in the field of data assimilation an increasing number of methodologies for the estimation of parameters of the prior error statistics are explicitly or implicitly based on this paradigm. One seminal article on the topic in data assimilation is the work of Dee [1995]. In our case, $p(\boldsymbol{\mu})$, the likelihood of the observation set, obtained as a normalization factor

$$
p(\boldsymbol{\mu})=\int \mathrm{d} \boldsymbol{\sigma} p(\boldsymbol{\mu} \mid \boldsymbol{\sigma}) p(\boldsymbol{\sigma}),
$$

is actually a hidden function of $r$ and $m$. We should write instead $p(\boldsymbol{\mu} \mid r, m)$. If they exist, the values of $r$ and $m$ that maximize $p(\boldsymbol{\mu} \mid r, m)$ are considered the most likely values of the parameters consistent with $\boldsymbol{\mu}$. One can seek for a numerical scheme that localize these values, for example the Desroziers's scheme, or we can screen the likelihood values for a large range of $(r, m)$ parameters.

[30] In the light of this discussion and early experiments, we have decided to use three hyperparameter estimation techniques. Each one of the three approaches is tested in the Gaussian case and semi-Gaussian case.

2.4.1. First Method: L-Curve and $\chi^{2}$

[31] The first approach makes use of the L-curve coupled to a $\chi^{2}$ diagnosis. The reason to use the L-curve technique is that it is also valid in the non-Gaussian context since it does not assume Gaussian statistics for the errors. However, this determines only one degree of freedom of the error statistics. In order to obtain the second one, we would like to tune the general level of errors in the system. If we note $J_{o}\left(\sigma_{a}\right)$ the degree of freedom for the noise defined by

$$
J_{o}\left(\boldsymbol{\sigma}_{a}\right)=\frac{1}{2}\left(\boldsymbol{\mu}-\mathbf{H} \boldsymbol{\sigma}_{a}\right)^{\mathrm{T}} \mathbf{R}^{-1}\left(\boldsymbol{\mu}-\mathbf{H} \boldsymbol{\sigma}_{a}\right)
$$

and $J_{b}\left(\sigma_{a}\right)$ the degree of freedom for the signal defined by

$$
J_{b}\left(\boldsymbol{\sigma}_{a}\right)=\frac{1}{2}\left(\boldsymbol{\sigma}_{a}-\boldsymbol{\sigma}_{b}\right)^{\mathrm{T}} \mathbf{B}^{-1}\left(\boldsymbol{\sigma}_{a}-\boldsymbol{\sigma}_{b}\right)
$$

then the quantity $2\left(J_{o}\left(\sigma_{a}\right)+J_{b}\left(\sigma_{a}\right)\right)$ should have the statistics of a $\chi^{2}$ if the prior errors are Gaussian, and as shown by Ménard et al. [2000], it should equal the number of observations when the prior statistics matches the genuine ones:

$$
J_{o}\left(\boldsymbol{\sigma}_{a}\right)+J_{b}\left(\boldsymbol{\sigma}_{a}\right)=\frac{d}{2} .
$$

where $d$ represents the number of observations. Note that in the semi-Gaussian case, the use of the $\chi^{2}$ diagnosis is necessarily approximate. The algorithm is as follows: Take a range of values for $r$ discretized into a set of values $r_{j}$. For any $r_{j}$ perform the L-curve analysis to get the optimal $m_{j}=m\left(r_{j}\right)$. Then, pick the right value of $r_{j}$, which satisfies the $\chi^{2}$ diagnosis for $r_{j}$ and $m_{j}$. 


\subsubsection{Second Method: Desroziers's Scheme}

[32] Under Gaussian assumptions, integral equation (10) can be computed analytically:

$$
p(\boldsymbol{\mu} \mid r, m)=\frac{e^{-\frac{1}{2}\left(\boldsymbol{\mu}-\mathbf{H} \sigma_{b}\right)^{\mathrm{T}}\left(\mathbf{R}+\mathbf{H B H} \mathbf{H}^{\mathrm{T}}\right)^{-1}\left(\boldsymbol{\mu}-\mathbf{H} \sigma_{b}\right)}}{\sqrt{(2 \pi)^{d}\left|\mathbf{R}+\mathbf{H B H}^{\mathrm{T}}\right|}} .
$$

[33] Our approach consists in trying to numerically maximize the likelihood. The log likelihood can be written:

$$
\begin{aligned}
\ln p(\boldsymbol{\mu} \mid r, m)= & -\frac{1}{2}\left(\boldsymbol{\mu}-\mathbf{H} \boldsymbol{\sigma}_{b}\right)^{\mathrm{T}}\left(\mathbf{R}+\mathbf{H B H}^{\mathrm{T}}\right)^{-1}\left(\boldsymbol{\mu}-\mathbf{H} \boldsymbol{\sigma}_{b}\right) \\
& -\frac{1}{2} \ln \left|\mathbf{R}+\mathbf{H B H ^ { \mathrm { T } }}\right|+C \\
= & -\frac{1}{2} \operatorname{tr}\left(\mathbf{S}_{r, m}^{-1} \overline{\mathbf{S}}\right)-\frac{1}{2} \ln \left|\mathbf{S}_{r, m}\right|+C,
\end{aligned}
$$

where $\mathbf{S}_{r, m}=\mathbf{R}+\mathbf{H B H}^{\mathrm{T}}$ is the prior statistics of the innovation, $\overline{\mathbf{S}}=\left(\boldsymbol{\mu}-\mathbf{H} \boldsymbol{\sigma}_{b}\right)\left(\boldsymbol{\mu}-\mathbf{H} \boldsymbol{\sigma}_{b}\right)^{\mathrm{T}}$ is the empirical statistics of the innovation, and $C$ is an irrelevant constant. The optimization of the log likelihood with respect to a parameter denoted $\lambda$, leads to the zero gradient condition

$$
0=\nabla_{\lambda} \ln p(\boldsymbol{\mu} \mid r, m)=\frac{1}{2} \operatorname{tr}\left[\left(\mathbf{S}_{r, m}^{-1} \overline{\mathbf{S}}-\mathbf{I}_{d}\right) \mathbf{S}_{r, m}^{-1} \nabla_{\lambda} \mathbf{S}_{r, m}\right] .
$$

Choosing $\lambda=r$ and $\lambda=m$, one obtains two scalar equations for the likely hyperparameters. These equations can be put in the form

$$
r^{2}=\frac{2 J_{o}^{\prime}\left(\boldsymbol{\sigma}_{a}\right)}{\operatorname{tr}\left(\mathbf{I}_{d}-\mathbf{H K}\right)}, \quad m^{2}=\frac{2 J_{b}^{\prime}\left(\boldsymbol{\sigma}_{a}\right)}{\operatorname{tr}(\mathbf{K H})},
$$

where

$$
\begin{gathered}
\mathbf{K}=\mathbf{B H}^{\mathrm{T}}\left(\mathbf{R}+\mathbf{H B H}^{\mathrm{T}}\right)^{-1}, \\
J_{o}^{\prime}(\boldsymbol{\sigma})=\frac{1}{2}(\boldsymbol{\mu}-\mathbf{H} \boldsymbol{\sigma})^{\mathrm{T}}(\boldsymbol{\mu}-\mathbf{H} \boldsymbol{\sigma}), \\
J_{b}^{\prime}(\boldsymbol{\sigma})=\frac{1}{2}\left(\boldsymbol{\sigma}-\boldsymbol{\sigma}_{b}\right)^{\mathrm{T}}\left(\boldsymbol{\sigma}-\boldsymbol{\sigma}_{b}\right) .
\end{gathered}
$$

Desroziers and Ivanov [2001] have shown that these two equations can be used as an iterative system which converges to a fixed point which could be a maximum of the likelihood [Chapnik et al., 2004]. They have shown that they could successfully be used in an operational meteorological model [Chapnik et al., 2006].

[34] Let us first remark that this scheme is consistent for Gaussian statistics, not necessarily for non-Gaussian ones. Second, this scheme, that we shall call Desroziers's scheme later on, converges to one local maximum, whereas there could be several maxima for the likelihood. The context in which we shall use this system is different from Chapnik et al. [2004], because their operator $\mathbf{H}$ is the observation operator, while ours represents the ATM model as well. As a consequence, $\mathbf{H B H}^{\mathrm{T}}$ partly populates off-diagonal terms of the innovation statistics $\mathbf{R}+\mathbf{H B H}^{\mathrm{T}}$ so that the sensitivity of the likelihood with respect to $r$ or $m$ should be sufficient to discriminate the two hyperparameters.

\subsubsection{Third Method: Unapproximated Maximum Likelihood Values Screening}

[35] The third method consists in estimating the exact value of the likelihood for a large range of $(r, m)$ parameters.

[36] In the Gaussian Case, we use the exact formula equation (14) to estimate the likelihood. With two hyperparameters and computer power, it is possible to perform a value screening of the likelihood, if the number of observations is limited.

[37] In the semi-Gaussian case, the Gaussian likelihood equation (14) is not valid anymore. We will numerically compute integral equation (10) in the semi-Gaussian case:

$$
\begin{aligned}
p(\boldsymbol{\mu} \mid r, m)= & \frac{1}{\sqrt{(2 \pi)^{d}|\mathbf{R}|(\pi / 2)^{N}|\mathbf{B}|}} \\
& \times \int_{\sigma \geq \mathbf{0}} \mathrm{d} \boldsymbol{\sigma} e^{-\frac{1}{2}(\boldsymbol{\mu}-\mathbf{H} \boldsymbol{\sigma})^{\mathrm{T}} \mathbf{R}^{-1}(\boldsymbol{\mu}-\mathbf{H} \boldsymbol{\sigma})-\frac{1}{2}\left(\boldsymbol{\sigma}-\boldsymbol{\sigma}_{b}\right)^{\mathrm{T}} \mathbf{B}^{-1}\left(\boldsymbol{\sigma}-\boldsymbol{\sigma}_{b}\right)} \\
= & \frac{e^{-\frac{1}{2}\left(\boldsymbol{\mu}-\mathbf{H} \boldsymbol{\sigma}_{b}\right)^{\mathrm{T}}\left(\mathbf{R}+\mathbf{H B H} \mathbf{H}^{\mathrm{T}}\right)^{-1}\left(\boldsymbol{\mu}-\mathbf{H} \sigma_{b}\right)}}{\sqrt{(2 \pi)^{d}|\mathbf{R}|(\pi / 2)^{N}|\mathbf{B}|}} \\
& \times \int_{\boldsymbol{\sigma} \geq \mathbf{0}} \mathrm{d} \boldsymbol{\sigma} e^{-\frac{1}{2}\left(\boldsymbol{\sigma}-\boldsymbol{\sigma}_{a}\right)^{\mathrm{T}} \mathbf{P}_{a}^{-1}\left(\boldsymbol{\sigma}-\boldsymbol{\sigma}_{a}\right)},
\end{aligned}
$$

where $\boldsymbol{\sigma}_{a}$ has been defined by equation (7) and $\mathbf{P}_{a}$ by equation (8). A Monte Carlo estimation of the integral term will be used. To do so, equation (21) can be written as:

$$
\begin{aligned}
p(\boldsymbol{\mu} \mid r, m)= & \frac{e^{-\frac{1}{2}\left(\boldsymbol{\mu}-\mathbf{H} \boldsymbol{\sigma}_{b}\right)^{\mathrm{T}}\left(\mathbf{R}+\mathbf{H B H} \mathbf{H}^{\mathrm{T}}\right)^{-1}\left(\boldsymbol{\mu}-\mathbf{H} \boldsymbol{\sigma}_{b}\right)}}{\sqrt{(2 \pi)^{d}\left|\mathbf{H B H} \mathbf{H}^{\mathrm{T}}+\mathbf{R}\right|}} \\
& \times 2^{N} \int_{\boldsymbol{\sigma} \geq 0} \frac{e^{-\frac{1}{2}\left(\boldsymbol{\sigma}-\boldsymbol{\sigma}_{a}\right)^{\mathrm{T}} \mathbf{P}_{a}^{-1}\left(\boldsymbol{\sigma}-\boldsymbol{\sigma}_{a}\right)}}{\sqrt{(2 \pi)^{N}\left|\mathbf{P}_{a}\right|}} \mathrm{d} \boldsymbol{\sigma},
\end{aligned}
$$

which can be seen as the product of the regular "Gaussian case" term by a correction taking into account the positivity of the source term. To estimate the value of the integral, we used the GHK simulator method from Hajivassiliou et al. [1996]. Our implementation is detailed in Appendix A.

[38] In total, six cases will be tested depending on whether the background statistics is assumed Gaussian or semiGaussian, and depending on the choice of the approaches (L-curve and $\chi^{2}$, Desroziers's scheme, and exact value screening of the likelihood).

\section{Application to the Fukushima Daiichi Accident}

\subsection{Observations}

[39] If all the radionuclides measurements of the International Monitoring System (IMS) of the Comprehensive Nuclear-Test-Ban Treaty Organization (CTBTO) have still not been released publicly, some activity concentrations measurements are available. In the United States, they come from the Radnet monitoring network which is supported by the Environmental Protection Agency (EPA) and from the CTBTO air monitoring stations (USP70 in California, USP77, USP78, USP79 and USP80 on islands in the Pacific Ocean), whose data have been publicly released by the United States National Data Center (USNDC). In Canada, the Health ministry has also released some monitoring measurement data. The Philippine Nuclear Research 
Table 1. Source of the Measurements of Activity Concentration for Cesium-137 and Iodine-131 Used in This Study

\begin{tabular}{lcc}
\hline \multicolumn{1}{c}{ Source } & Cesium-137 & Iodine-131 \\
\hline Japan & & \\
KEK-NIES, Tsukuba & 10 & 10 \\
MITRI, Tokyo & 58 & 160 \\
CPDNP, JPP38 CTBTO station & 20 & 9 \\
MEXT, Fukushima Prefecture & 16 & 54 \\
Total Japan & 104 & 233 \\
Outside Japan & 77 & 82 \\
USNDC, CTBTO, USA & 44 & 69 \\
EPA, RadNet, USA & 29 & 31 \\
Health Ministry Of Canada & 13 & 13 \\
PNRI, PHP52 CTBTO station, & & \\
$\quad$ Philippines & 163 & 195 \\
Total Outside & 267 & 428 \\
Total &
\end{tabular}

Institute (PNRI) has displayed the measurements from the CTBTO air monitoring station PHP52 on its website. In Japan, the High Energy Accelerator Research Organization (KEK) and the National Institute for Environmental Studies (NIES), located in Tsukuba, and the Metropolitan Industrial Technology Research Institute (MITRI), in Tokyo, have performed some measurements. In Fukushima prefecture, the Ministry of Education, Culture, Sport, Science and Technology (MEXT) has performed a large number of measurements. Because the Eulerian atmospheric transport model in this study is used at a spatial resolution of $0.25^{\circ}$, we have excluded measurements within a radius of $30 \mathrm{~km}$ around the power plant. Finally, the Center for the Promotion of Disarmament and Non-Proliferation (CPDNP) has released the measurements from the JPP38 CTBTO air monitoring station. Altogether, 267 measurements of cesium-137 activity concentration (104 in Japan and 163 outside) and 428 measurements of iodine-131 activity concentration (233 in Japan and 195 outside) are assimilated in this study (see Table 1 and Figure 1). The iodine measurements should be understood as a lower bound, since only the aerosol form may be collected in particle filters.

\subsection{Atmospheric Transport Model}

[40] The simulations of the dispersion of radionuclides from Daiichi nuclear power plant have been performed with the chemistry transport model Polair3D, the Eulerian model of the Polyphemus platform. As far as radionuclides are concerned, it has been validated on the European Tracer Experiment, on the Algeciras incident and on the Chernobyl accident [Quélo et al., 2007].

[41] The model integrates the concentration field $c$ of either ${ }^{137} \mathrm{Cs}$ or ${ }^{131} \mathrm{I}$, following the transport equation

$$
\frac{\partial c}{\partial t}+\operatorname{div}(\boldsymbol{u} c)=\operatorname{div}\left(\rho \boldsymbol{K} \nabla\left(\frac{c}{\rho}\right)\right)-\Lambda^{\mathrm{s}} c-\Lambda^{\mathrm{d}} c+\sigma
$$

where $\Lambda^{\mathrm{s}}$ is the wet scavenging rate, $\Lambda^{\mathrm{d}}$ represents the radioactive decay and $\sigma$ is the point-wise source for the radionuclide. $\boldsymbol{K}$ is the matrix of turbulent diffusion, diagonal in practice. The vertical component is given by $K_{z}$, computed with Louis parameterization [Louis, 1979]. The horizontal component $K_{H}$ is taken constant (equal to $25,000 \mathrm{~m}^{2}$ $\mathrm{s}^{-1}$ for a spatial resolution of $0.25^{\circ}$ ). Except for the boundary condition on the ground, all boundary conditions are taken null. The boundary condition on the ground is

$$
-K_{z} \nabla c \cdot \mathbf{n}=-v^{\mathrm{dep}} c
$$

where $\mathbf{n}$ is the unitary vector upward oriented, and $v^{\mathrm{dep}}$ is the dry deposition velocity of ${ }^{137} \mathrm{Cs}$ or ${ }^{131} \mathrm{I}$.

[42] The advection is implemented thanks to a third-order direct space-time scheme, with a Koren-Sweby flux limiter function. Because of the sharp gradients found, it is important that such a limiter be used. The diffusion scheme is integrated through an implicit second-order Rosenbrock scheme, with a three-point spatial scheme, and directional splitting.

[43] Cesium-137 and iodine-131 are modeled as passive gaseous tracers with radioactive decay. Their half-lives are 11,000 and 8.04 days, respectively. Dry deposition is modeled by using a simple scheme with a constant deposition velocity: $v^{\mathrm{dep}}=0.2 \mathrm{~cm} \mathrm{~s}^{-1}$ for ${ }^{137} \mathrm{Cs}$ and $v^{\mathrm{dep}}=0.5 \mathrm{~cm} \mathrm{~s}^{-1}$ for ${ }^{131} \mathrm{I}$. As far as the wet scavenging is concerned, the parameterization used in this study is of the form:

$$
\left\{\begin{array}{l}
\Lambda^{\mathrm{s}}=0 \quad \text { if } R H<R H_{t} \\
\Lambda^{\mathrm{s}}=3.5 \times 10^{-5}\left(\frac{R H-R H_{t}}{R H_{s}-R H_{t}}\right) \quad \text { otherwise }
\end{array}\right.
$$
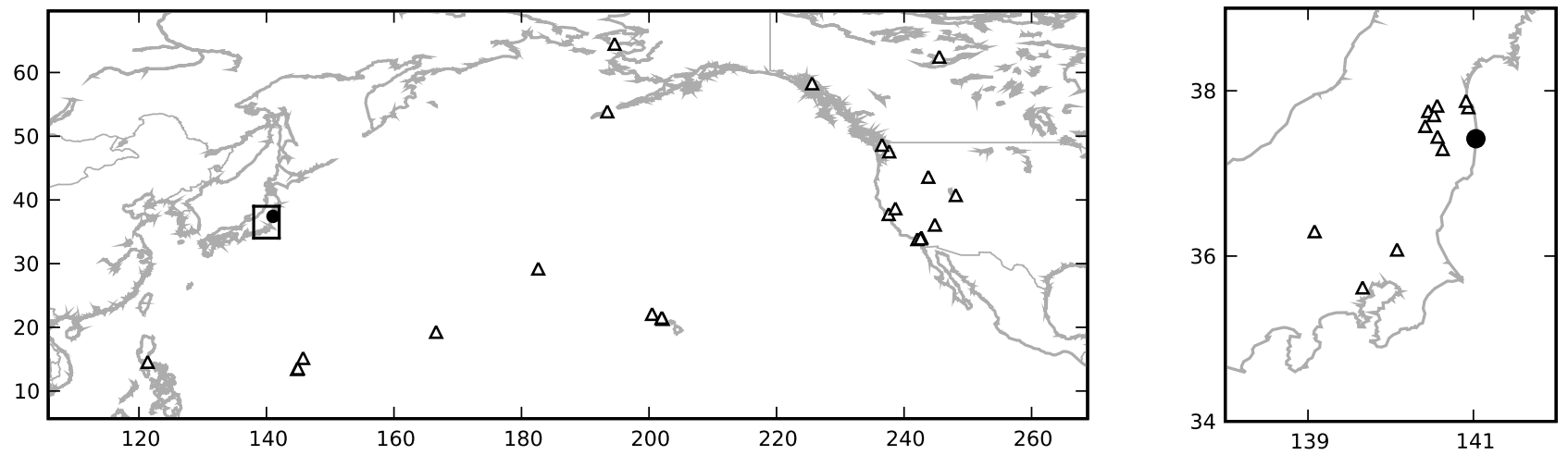

Figure 1. (left) Map of the simulation domain used in Polair3D. The triangles show the observations locations. The circle represents the Fukushima Daiichi power plant. (right) Map of boxed area over Japan and the observations sites available over the country. 
Table 2. Estimation of Parameters and Corresponding Reconstructed Released Activity With the Different Methods in Gaussian Case for Cesium-137 Source Reconstruction

\begin{tabular}{|c|c|c|c|}
\hline Parameter & Method & With Observations in Japan (104) & With All Observations (267) \\
\hline \multirow[t]{3}{*}{$r\left(\mathrm{~Bq} \mathrm{~m}^{-3}\right)$} & $\chi^{2}+$ L-curve & 1.05 & 1.03 \\
\hline & Desroziers's scheme & 1.0 & 0.56 \\
\hline & Maximum likelihood & 1.0 & 0.56 \\
\hline \multirow[t]{3}{*}{$m\left(\mathrm{~Bq} \mathrm{~s}^{-1}\right)$} & $\chi^{2}+$ L-curve & $1.5 \times 10^{12}$ & $1.5 \times 10^{12}$ \\
\hline & Desroziers's scheme & $1.5 \times 10^{12}$ & $2.2 \times 10^{12}$ \\
\hline & Maximum likelihood & $1.5 \times 10^{12}$ & $2.2 \times 10^{12}$ \\
\hline \multirow[t]{3}{*}{ Released activity (Bq) } & $\chi^{2}+$ L-curve & $2.8 \times 10^{16}$ & $6.9 \times 10^{16}$ \\
\hline & Desroziers's scheme & $2.8 \times 10^{16}$ & $2.1 \times 10^{17}$ \\
\hline & Maximum likelihood & $2.8 \times 10^{16}$ & $2.1 \times 10^{17}$ \\
\hline
\end{tabular}

where $R H$ stands for the relative humidity, $R H_{t}$ being a threshold value $(=80 \%)$ and $R H_{s}$ is the saturation value (=100\%) [Pudykiewicz, 1989; Brandt et al., 2002].

[44] The domain of simulation is an extended domain covering Japan, the North Pacific Ocean and an important part of the North American continent, with a spatial resolution of $0.25^{\circ} \times 0.25^{\circ}$. The number of grid points in the domain simulation is $652 \times 256$. The Polair3D model is configured with 15 vertical levels ranging from 0 to $8000 \mathrm{~m}$.

[45] This study attempts to reconstruct the source term from 11 March to 26 March (which represents 384 one hour time steps). However, the simulations run over a longer period (from 11 March to 5 April) in order to exploit the information content of later observations. A total of 384 direct simulations are performed to fill the Jacobian matrix $\mathbf{H}$ column by column, and no adjoint model is needed in this process [see Winiarek et al., 2011]. The meteorological fields used in this study are the fields computed by the operational model from the European Centre for MediumRange Weather Forecasts (ECMWF). They have a resolution of $0.25^{\circ} \times 0.25^{\circ}$ and are available every $3 \mathrm{~h}$.

\subsection{Gaussian Case and Cesium-137}

[46] Observations collected in Japan and those collected elsewhere are of different nature. First, the distances between the Daiichi power plant and the monitoring stations are so different that they induce a large gap in the order of magnitude of the observation values. All observations in Japan are in the range $\left[10^{-1}, 10^{2}\right] \mathrm{Bq} \mathrm{m}^{-3}$ whereas most observations outside Japan are in the range $\left[10^{-6}, 10^{-3}\right] \mathrm{Bq}$ $\mathrm{m}^{-3}$. Impacts of the model errors (meteorological fields and loss process parameterizations) accumulate along this longdistance transport path. Additionally, most of observations outside Japan are mean measurements over a long period (typically $24 \mathrm{~h}$ ), whereas in Japan measurements are typically representative of shorter periods ( $1 \mathrm{~h}$ or less). For these reasons, we have chosen to perform first the inversions using only the Japanese observations, and then using all the available observations to study the impact of those differences.

\subsubsection{Estimation of Parameters and Total Released Activity}

[47] Under Gaussian assumptions for both observation and background errors statistics, the three methods are comparable. Estimates for $r, m$ and the total released activity are reported in Table 2.
[48] In particular we have verified that under Gaussian assumptions, Desroziers's scheme exactly converges to the values $(r, m)$ given by the maximum likelihood method. The value screening of the likelihood (see Figure 2) allows to plot the marginal posterior distribution function for both parameters $r$ and $m$ (see Figures 3 and 4) on which two local maxima appear. Given our initial couple of parameters, Desroziers's scheme converges toward the absolute maximum location. (With a different initial condition in another basin of attraction, it could have converged to the other.)

[49] The reconstructed total mass of ${ }^{137} \mathrm{Cs}$ released in the atmosphere lies between $2.8 \times 10^{16} \mathrm{~Bq}$ and $2.1 \times 10^{17} \mathrm{~Bq}$, which is consistent, though slightly higher, with the first estimates released by the Nuclear Safety Commission of Japan $\left(1.2 \times 10^{16} \mathrm{~Bq}\right.$ at first, then $1.3 \times 10^{16} \mathrm{~Bq}$ from Chino et al. [2011]).

\subsubsection{Temporal Profile and Uncertainty Reduction}

[50] Most of the time, the meteorological conditions have transported the radionuclides toward the Pacific Ocean, sparing populations, but reducing the observability of the plume. As a consequence, the inversion methods are only able to reconstruct some time windows when attempting to

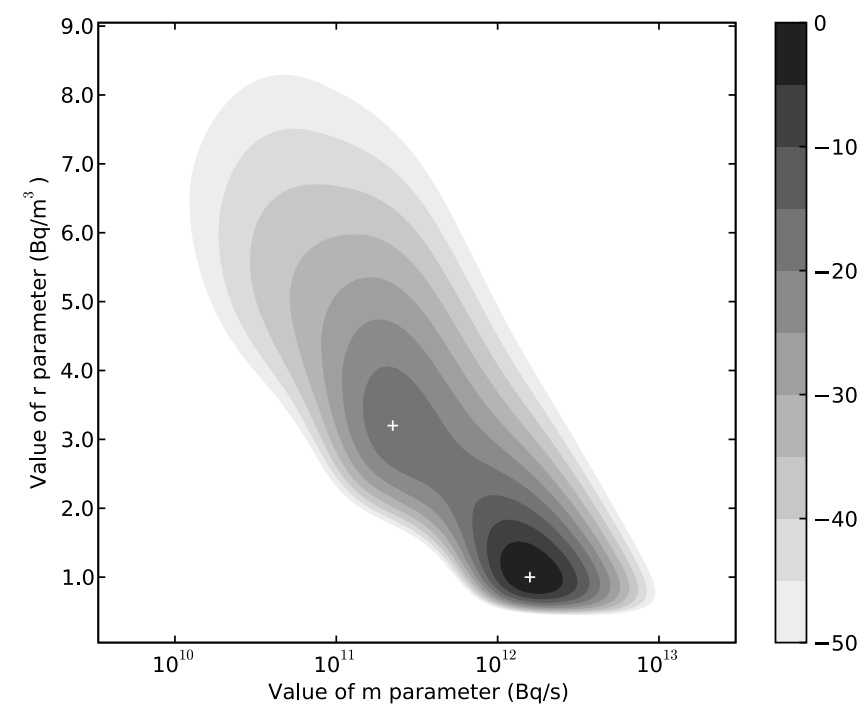

Figure 2. Density plot of the log likelihood under Gaussian assumptions. The global maximum log likelihood value has been subtracted to all log likelihood values, so that the maximum value is set to zero. 


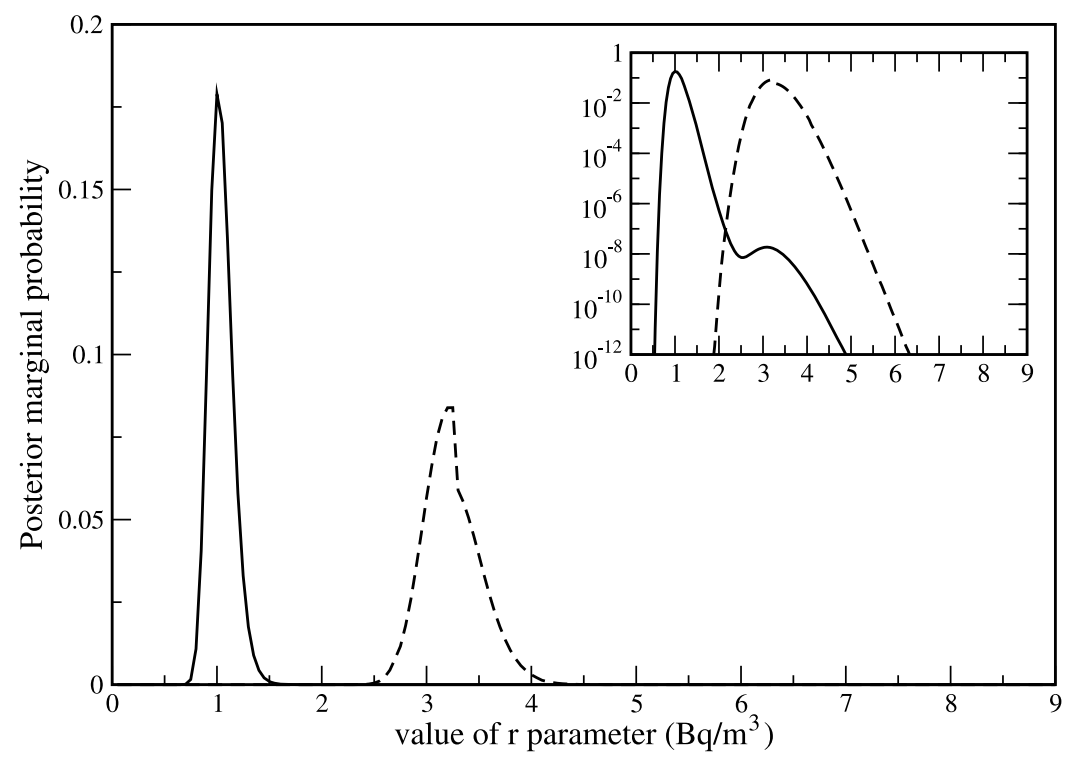

Figure 3. Marginal probability density function (pdf) plot of parameter $r$ obtained with the maximum likelihood method under Gaussian assumptions (full line) or semi-Gaussian assumptions (dashed line) with the Japanese data of cesium-137 activity concentrations. The inset displays the same pdfs in logarithmic scale.

retrieve the temporal profile of the source term. The profile of the reconstructed source using the set of observations over Japan is displayed in Figure 5. In particular three periods are well observed: the first one lies approximatively from 14 March to 16 March, the second one from 19 March to 21 March and the last one from 24 March to 26 March, when winds were blowing in the southwest direction, where the monitoring stations of Tokyo, Tsukuba and JPP38 are located.

[51] Because of the Gaussian assumptions, the source parameters are allowed to take negative values. From
Figure 5, it is clear that the number of observations is not sufficient to constrain the solution to be positive for most parameters. A larger number of observations would have probably helped in that respect as shown by Winiarek et al. [2011]. Consequently, in the absence of a large number of observations, non-Gaussian statistical assumptions that constrain the source to be positive are paramount.

[52] In Figure 5, the uncertainty (standard deviation) of the retrieved source parameter is also plotted. It has been computed using the diagonal parameters of $\mathbf{P}_{a}$, which is

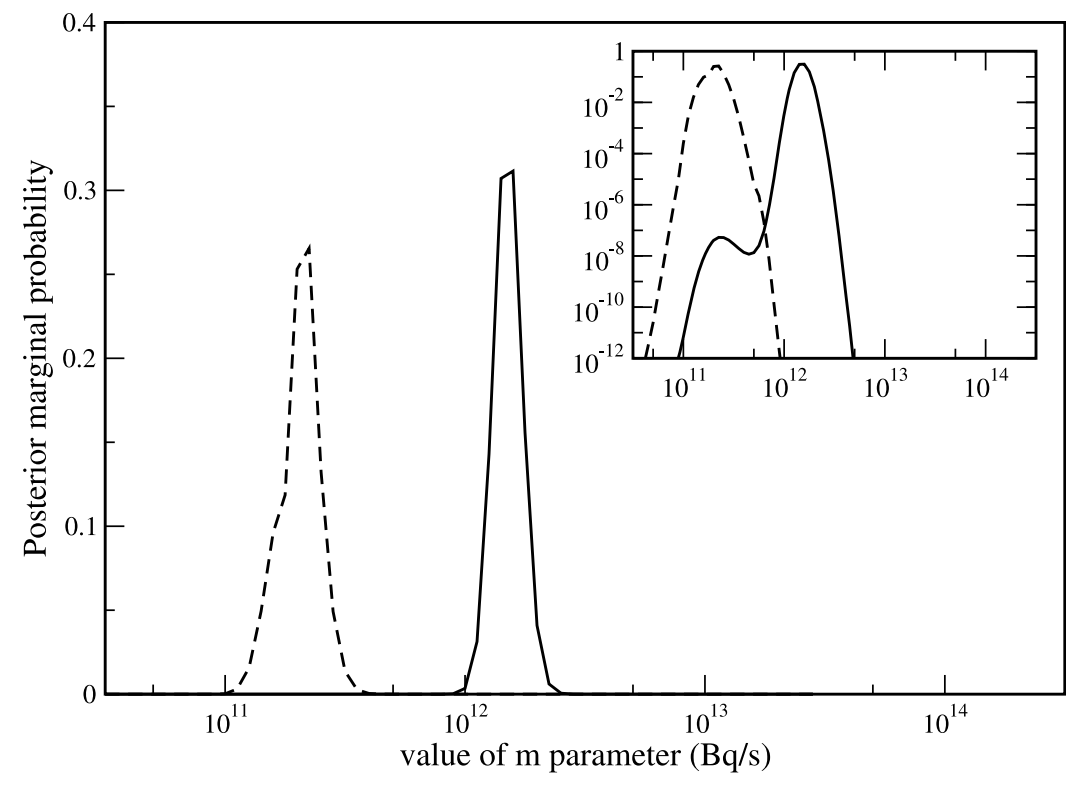

Figure 4. Marginal pdf of parameter $\mathrm{m}$ obtained with the maximum likelihood method under Gaussian assumptions (full line) or semi-Gaussian assumptions (dashed line) with the Japanese data of cesium-137 activity concentrations. The inset displays the same pdfs in logarithmic scale. 


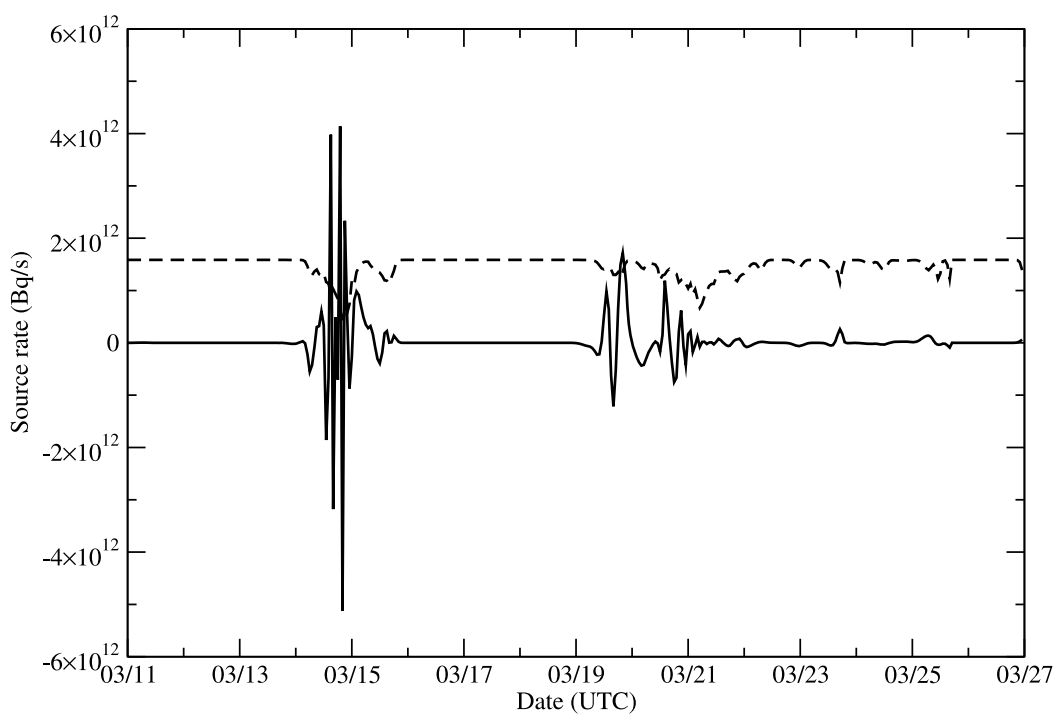

Figure 5. Reconstructed temporal profile of cesium-137 under Gaussian assumptions with the maximum likelihood method (equivalent to Desroziers's scheme), using the observations of Japan. The dashed line represents the posterior uncertainty on the reconstructed source (diagonal terms of matrix $\mathbf{P}_{a}$ ).

an approximation since it neglects the correlations between parameters.

\subsection{Semi-Gaussian Case and Cesium-137}

\subsubsection{Estimation of Parameters and Total}

\section{Released Activity}

[53] Under semi-Gaussian assumptions for the background errors statistics, the three methods are expected to yield more distinct results. These results are displayed in Table 3. The $\chi^{2}$ diagnosis, as well as the Desroziers's scheme, are based on Gaussian assumptions and this is why the first two methods are to be considered approximations in this case. Nevertheless, results obtained with these two methods are comparable to those given by the value screening of the likelihood (which is unapproximated), especially when more observations are assimilated. The posterior marginal pdf of $r$ and $m$ parameters are also displayed in Figures 3 and 4. In the situation where all available data are used, the total released activity of ${ }^{137} \mathrm{Cs}$ released is estimated to be between $1.0 \times 10^{16} \mathrm{~Bq}$ and $1.9 \times 10^{16} \mathrm{~Bq}$. Focusing on the most reliable methods $\left(\chi^{2}+\right.$ L-curve and maximum likelihood), and only assimilating observations over Japan to avoid large model errors, we obtain an estimation of $1.2 \times 10^{16} \mathrm{~Bq}$.

3.4.2. Temporal Profile and Uncertainty Reduction

[54] As indicated in section 2.3, we have performed a second-order Monte Carlo analysis to estimate the posterior analysis error instead of using equation (8), which is only consistent (though approximate) with Gaussian statistics. In Figure 6 the reconstructed source using the set of observations over Japan, is displayed as well as its standard deviation computed from this Monte Carlo analysis. The same three periods of observability as in the Gaussian case are visible, but may be slightly enlarged. Even if the available data (activity concentration data as used in this study, but also deposition data and gamma dose rate within the NPP site) clearly indicate releases between 19 March and $21 \mathrm{March}$, it may be surprising to retrieve such a high peak on 20 March, as an emergency cooling process has been engaged using Tokyo Fire Department equipment since approximatively 18 March, as debated in the discussion manuscript of Stohl et al. [2011] and its subsequent comments. However, it can be seen on Figure 6 that a high uncertainty, estimated by our Monte Carlo analysis, is

Table 3. Estimation of Parameters and Corresponding Reconstructed Released Activity With the Different Methods in SemiGaussian Case for Cesium-137 Source Reconstruction

\begin{tabular}{|c|c|c|c|}
\hline Parameter & Method & With Observations in Japan (104) & With All Observations (267) \\
\hline \multirow[t]{3}{*}{$r\left(\mathrm{~Bq} \mathrm{~m}^{-3}\right)$} & $\chi^{2}+$ L-curve & 4.55 & 2.88 \\
\hline & Desroziers's scheme & 5.41 & 2.96 \\
\hline & Maximum likelihood & 3.25 & 1.7 \\
\hline \multirow[t]{3}{*}{$m\left(\mathrm{~Bq} \mathrm{~s}^{-1}\right)$} & $\chi^{2}+$ L-curve & $3.2 \times 10^{11}$ & $2.0 \times 10^{11}$ \\
\hline & Desroziers's scheme & $5.3 \times 10^{10}$ & $1.3 \times 10^{11}$ \\
\hline & Maximum likelihood & $2.0 \times 10^{11}$ & $3.5 \times 10^{11}$ \\
\hline \multirow[t]{3}{*}{ Released activity (Bq) } & $\chi^{2}+$ L-curve & $1.2 \times 10^{16}$ & $1.3 \times 10^{16}$ \\
\hline & Desroziers's scheme & $3.3 \times 10^{15}$ & $1.0 \times 10^{16}$ \\
\hline & Maximum likelihood & $1.2 \times 10^{16}$ & $1.9 \times 10^{16}$ \\
\hline
\end{tabular}




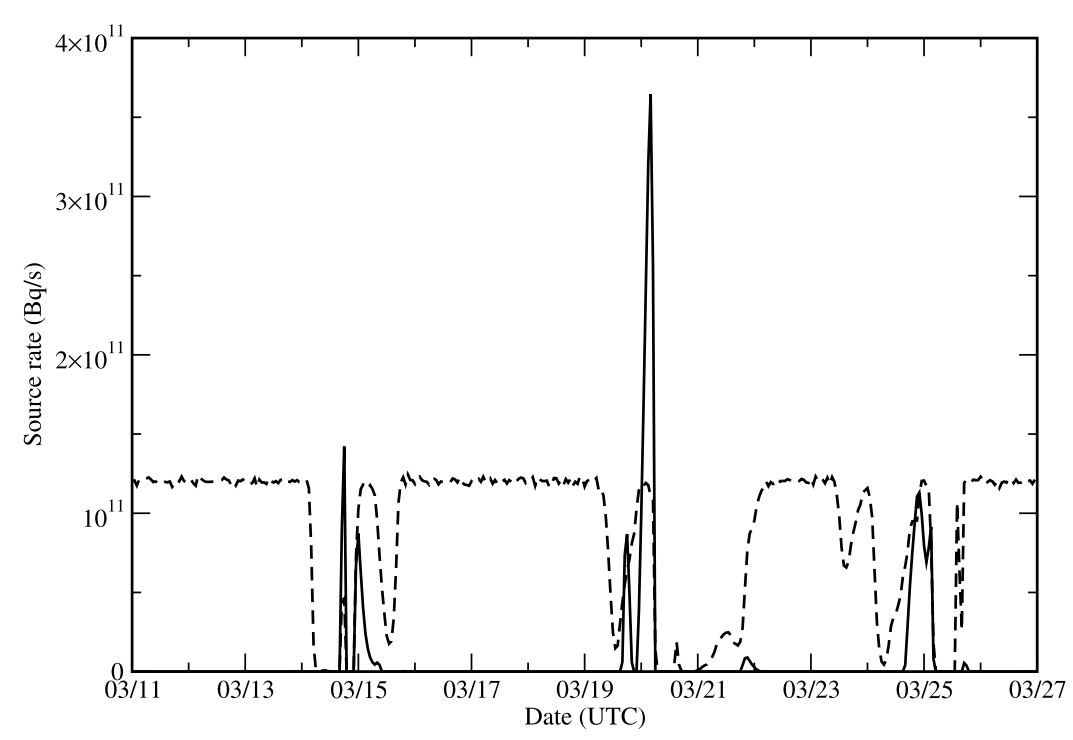

Figure 6. Reconstructed temporal profile of cesium-137 under semi-Gaussian assumptions with the maximum likelihood method, using the observations over Japan. The dashed line represents the standard deviation yielded by the Monte Carlo analysis.

attached to the magnitude of this peak. Moreover, from the end of this peak to 22 March, the retrieved emissions are weak and this is supported by a low uncertainty.

[55] The plume concentration field that is simulated using the source term obtained from all available observations and the maximum likelihood method is displayed in Figure 7. The activity concentration fields of cesium-137 are displayed at four different dates from 15 March to 21 March. In Figure 8 the comparison between simulated concentrations and observations is plotted at the MITRI station in Tokyo. The agreement between the simulation and the observations is good, especially at peak times, in spite of a time offset for the last peak (between 2 and $4 \mathrm{~h}$ ).

\subsection{Application to Iodine-131}

[56] In the case of iodine-131, we only consider the nonGaussian case since it was shown to have a significant advantage over the Gaussian methodology for cesium-137.

[57] The results are reported in Table 4 . With all the available observations, the total reconstructed released activity lies between $1.9 \times 10^{17} \mathrm{~Bq}$ and $7.0 \times 10^{17} \mathrm{~Bq}$. This estimate is of the same order of magnitude as the NSC first official estimates, although higher $\left(1.5 \times 10^{17} \mathrm{~Bq}\right.$ from Chino et al. [2011]).

[58] Focusing on the most reliable methods $\left(\chi^{2}+\right.$ L-curve and maximum likelihood), and only assimilating observations
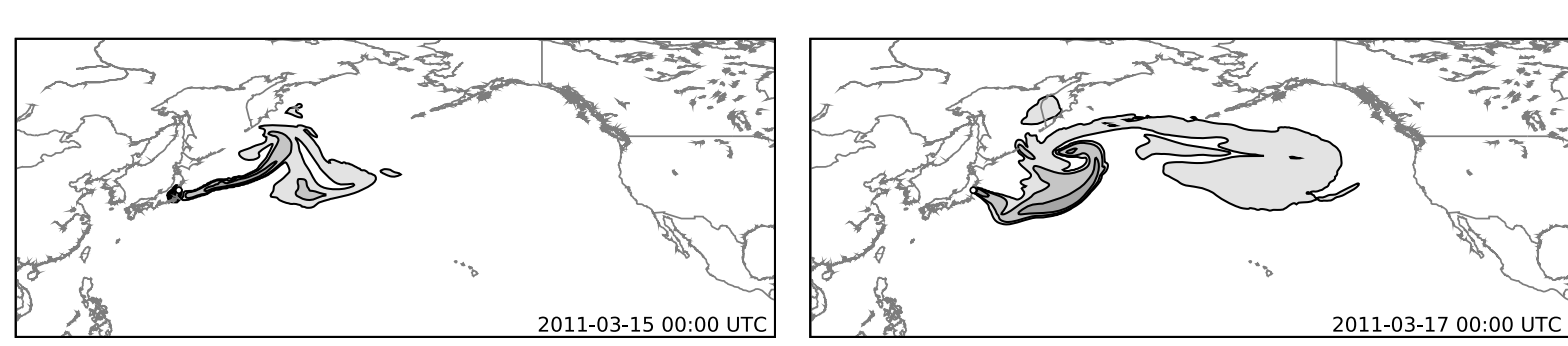

$10^{3}$
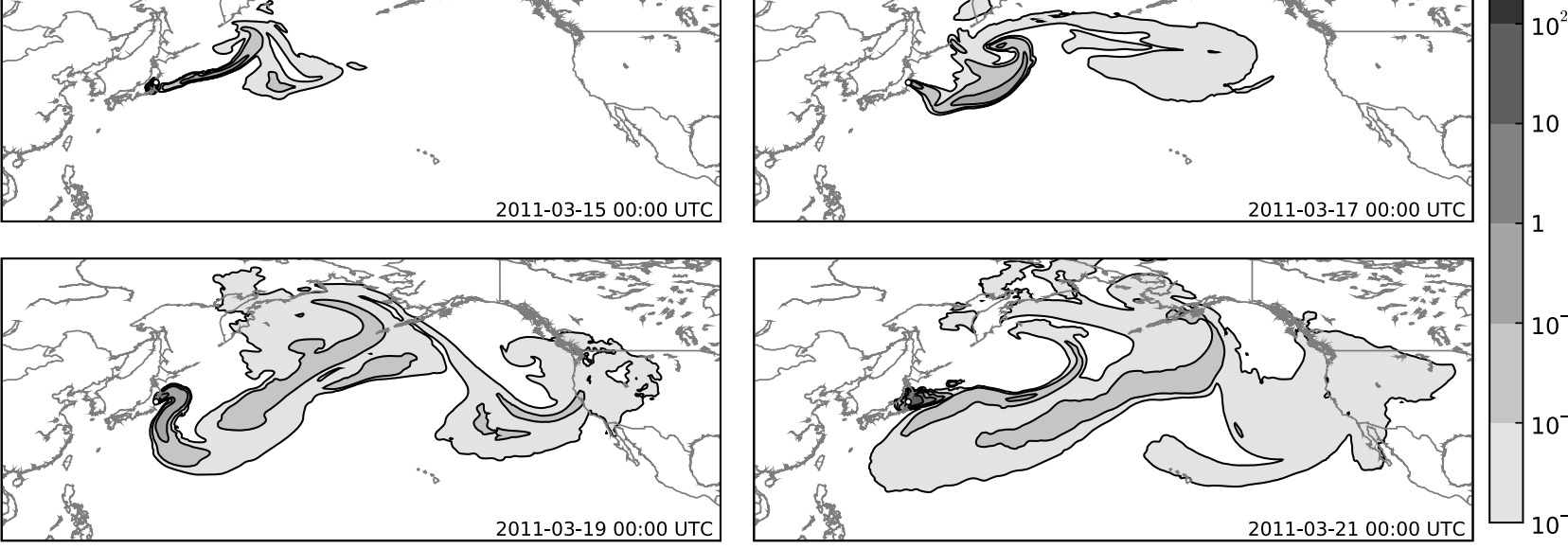

10

Figure 7. Map (in $\mathrm{Bq} \mathrm{m}^{-3}$ ) of simulated cesium-137 plume dispersion at four different dates after the accident start. The source term is the one reconstructed with all available data and the maximum likelihood method. 


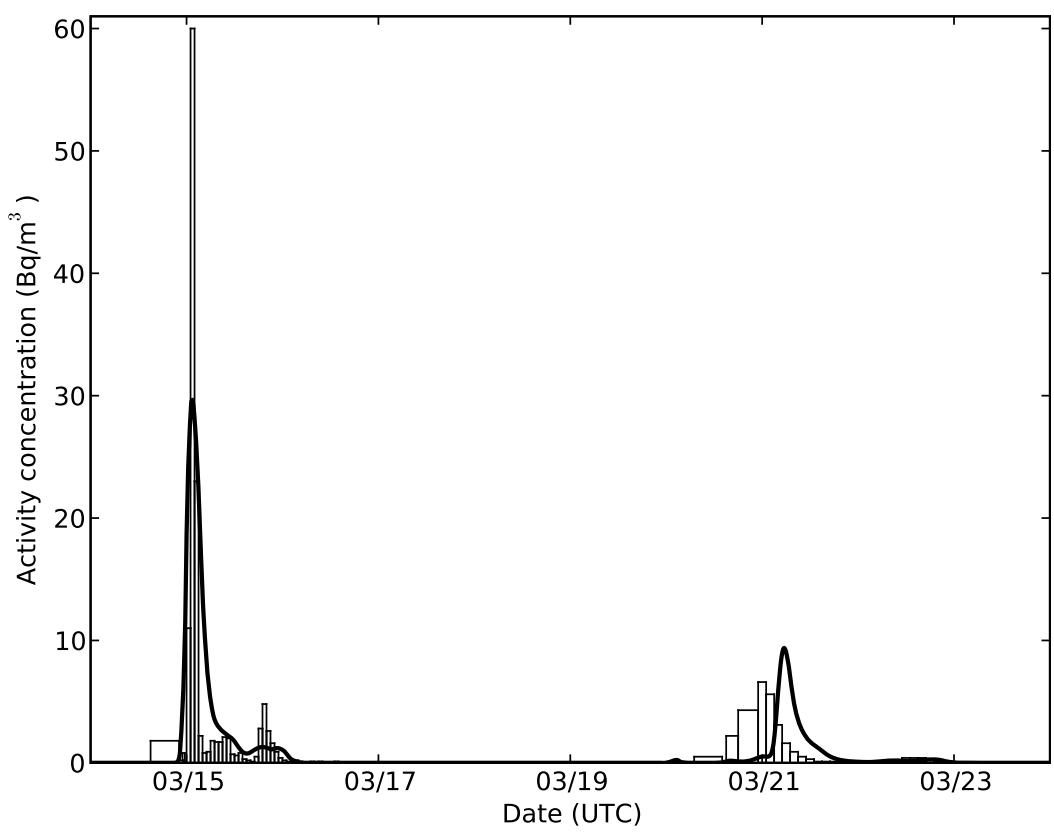

Figure 8. Comparison between simulated concentrations of cesium-137 and measurements data in Tokyo station (MITRI). The full line stands for the simulated concentrations using the source term provided by the maximum likelihood method. The histogram represents the observations. The width of the bars represents the duration of the measurements.

over Japan to avoid large model errors, we obtain estimates of $1.9 \times 10^{17} \mathrm{~Bq}$ and $3.8 \times 10^{17} \mathrm{~Bq}$, respectively.

[59] Similarly to the non-Gaussian case for cesium-137, the reconstruction profile when assimilating observations over Japan is reported in Figure 9. An estimation of the uncertainty of the retrieval obtained from Monte Carlo analysis is also plotted. Compared to cesium-137, there is a better observability from 19 March until 26 March without interruption. The reduction of uncertainty during this period is explained by a larger amount of available data, especially in Tokyo, MITRI station.

[60] As for cesium-137, a cartoon of the dispersion plume between 15 March and 21 March is displayed in Figure 10. The simulation is based on the iodine-131 source term estimated using all observations. In Figure 11 the comparison between simulated concentrations and observations is plotted at the MITRI station in Tokyo. The agreement between the simulation and the observations is good, especially at peak times.

\subsection{Uncertainty and Sensitivity Analysis}

[61] In this section, several key issues pertaining to the uncertainty of the prior assumptions, and of the retrievals are discussed.

\subsubsection{Quantitative Results and Uncertainties}

[62] The quantitative results are summarized in Table 5: the range of estimates using all observations and all methods, and the robust range of estimates, using Japanese observations and (more robust) non-Gaussian methods.

[63] In addition, the estimated standard deviations for the total retrieved activities are computed and reported in Table 5. It provides an estimation of the uncertainty attached to the robust result. Two standard deviations are computed. The first one results from the perturbation of the observations only. Therefore it is a measure of the uncertainty attached to the retrieved mass. A second one results from the perturbation of both the observations and the background. It is a measure of the expected total uncertainty that includes the releases that could not be observed. Obviously it is very

Table 4. Estimation of Parameters and Corresponding Reconstructed Released Activity With the Different Methods in Semi-Gaussian Case for Iodine-131 Source Reconstruction

\begin{tabular}{|c|c|c|c|}
\hline Parameter & Method & With Observations in Japan (233) & With All Observations (428) \\
\hline$r\left(\mathrm{~Bq} \mathrm{~m}^{-3}\right)$ & $\begin{array}{l}\chi^{2}+\text { L-curve } \\
\text { Desroziers's scheme } \\
\text { Maximum likelihood }\end{array}$ & $\begin{array}{l}14.0 \\
18.4 \\
5.60\end{array}$ & $\begin{array}{l}10.5 \\
10.6 \\
4.01\end{array}$ \\
\hline$m\left(\mathrm{~Bq} \mathrm{~s}^{-1}\right)$ & $\begin{array}{l}\chi^{2}+\text { L-curve } \\
\text { Desroziers's scheme } \\
\text { Maximum likelihood }\end{array}$ & $\begin{array}{l}2.7 \times 10^{12} \\
2.0 \times 10^{11} \\
5.6 \times 10^{12}\end{array}$ & $\begin{array}{l}2.0 \times 10^{12} \\
1.9 \times 10^{12} \\
7.1 \times 10^{12}\end{array}$ \\
\hline Released activity $(\mathrm{Bq})$ & $\begin{array}{l}\chi^{2}+\text { L-curve } \\
\text { Desroziers's scheme } \\
\text { Maximum likelihood }\end{array}$ & $\begin{array}{l}1.9 \times 10^{17} \\
1.6 \times 10^{16} \\
3.8 \times 10^{17}\end{array}$ & $\begin{array}{l}2.0 \times 10^{17} \\
1.9 \times 10^{17} \\
7.0 \times 10^{17}\end{array}$ \\
\hline
\end{tabular}




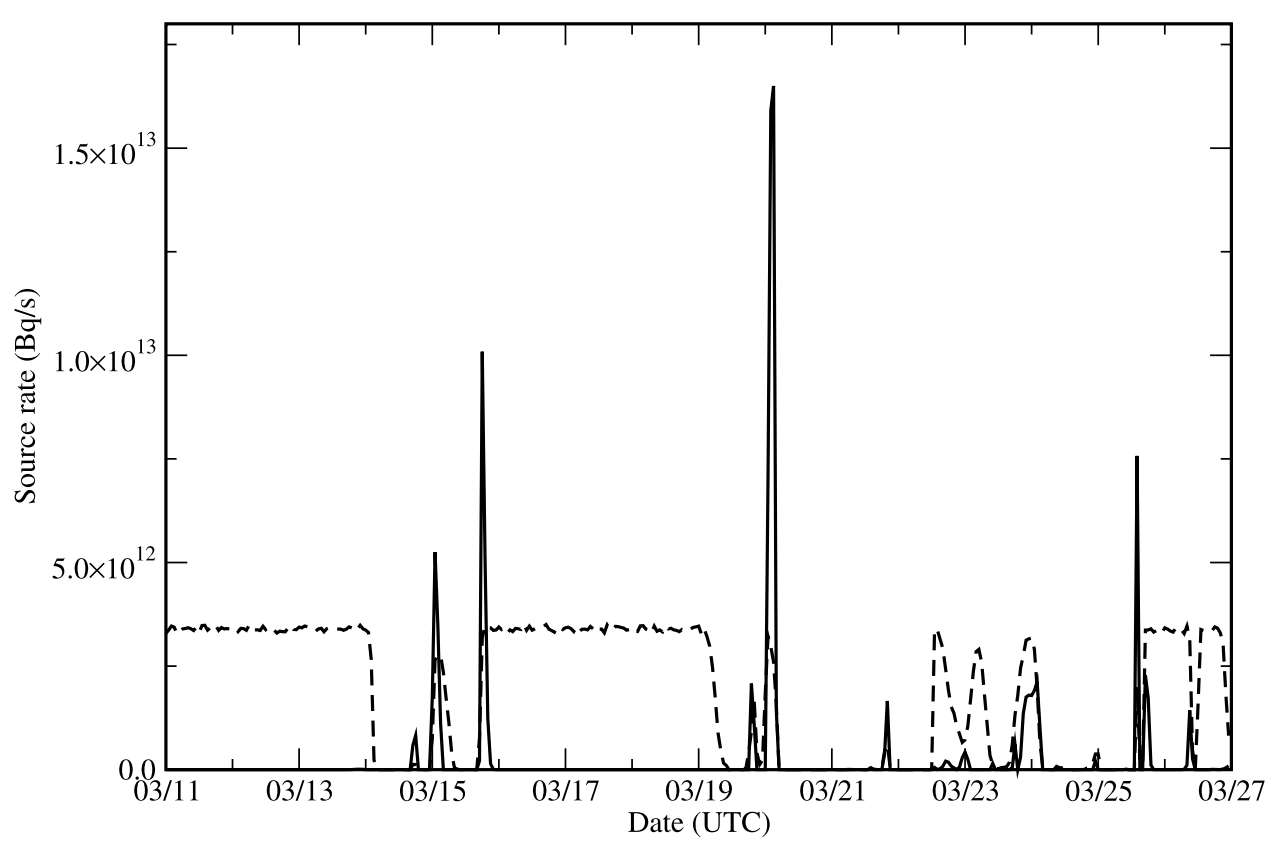

Figure 9. Reconstructed temporal profile of iodine-131 under semi-Gaussian assumptions with maximum likelihood method, using observations over Japan. The dashed line represents the standard deviation given by the Monte Carlo analysis.

speculative since it relies on the estimation of the background parameter $m$ for the whole period but which has been determined with observations that only shed light on a few days in the period.

[64] In Figure 12, the temporal profiles of the ${ }^{137} \mathrm{Cs}$ source term retrieved using only the observations in Japan on one hand, and all available observations on the other hand, are plotted. The use of all observations does not introduce new peaks in the profile, and the order of magnitude of the total released activity is unchanged. Nevertheless, significant emissions rates are retrieved during several periods (from 11 March to 14 March, from 16 March to 19 March and on
26 March), although at a much lower magnitude compared to the peaks of $15 \mathrm{March}$ and $20 \mathrm{March}$. The smoother shape of the distant observations contribution is due to the diffusive nature of atmospheric transport over such long distances, in addition to their longer duration. The total retrieved activity of this distant observations contribution only represents about $1 \%$ of the total retrieved activity.

\subsubsection{Background Uncertainty}

[65] As argued in the introduction, following Bocquet [2005] and Davoine and Bocquet [2007] and differently from standard practice in greenhouse gas flux inversions and atmospheric chemistry emission inversion where an
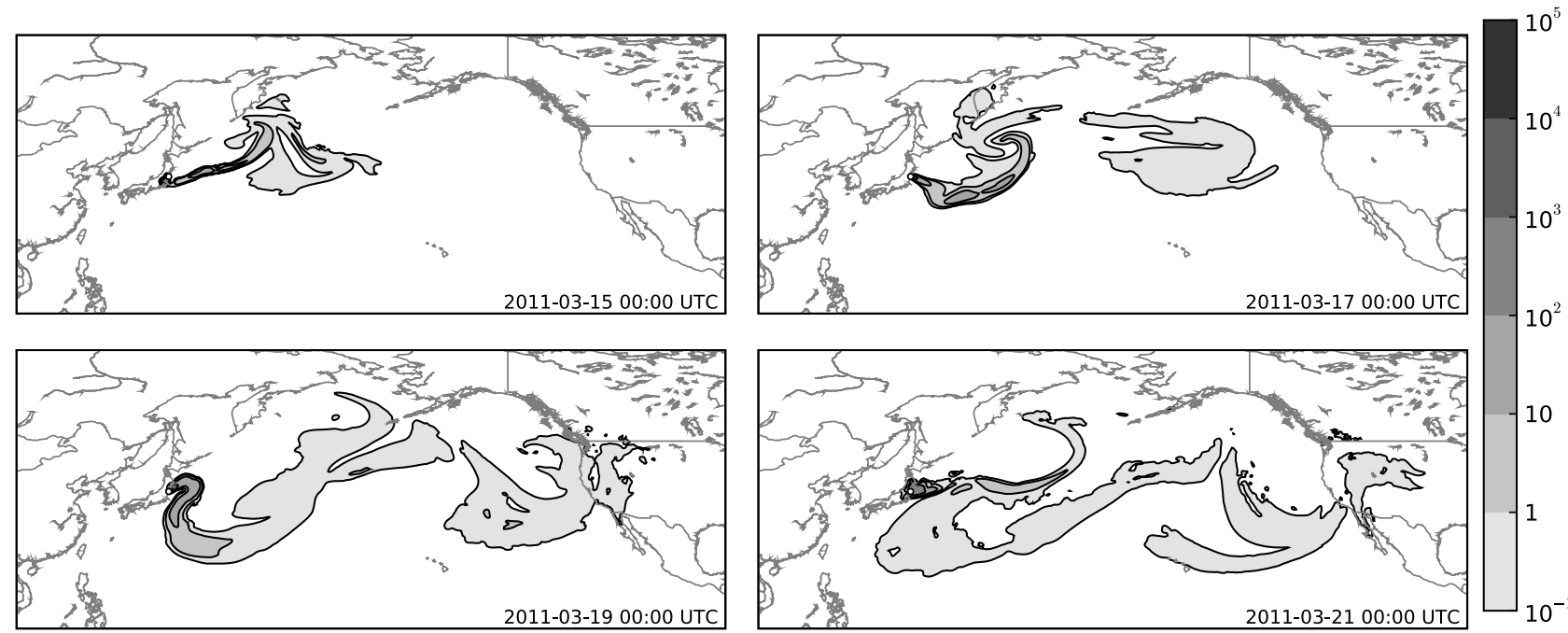

Figure 10. Map (in $\mathrm{Bq} \mathrm{m}^{-3}$ ) of simulated iodine-131 plume dispersion at four different dates after the accident start. The source term is the one reconstructed with all available data and the maximum likelihood method. 


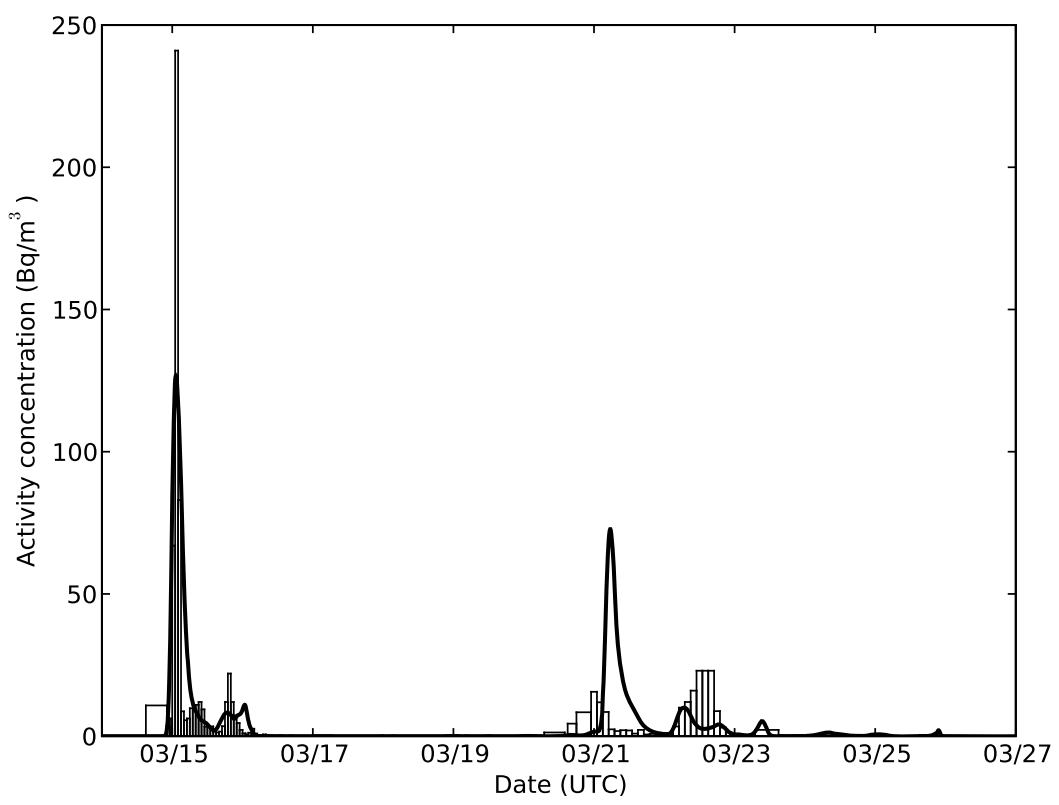

Figure 11. Comparison between simulated concentrations of iodine-131 and measurements data in Tokyo station (MITRI). The full line stands for the simulated concentrations using the source term provided by the maximum likelihood method. The histogram represents the observations. The width of the bars represents the duration of the measurements.

inventory is often available, we have chosen a first guess (background term) equal to zero. In our opinion, it particularly suits the context of the Fukushima Daiichi accident. Indeed the emission has been intermittent (strong emission peaks occur at the time of hydrogen explosions or during the ventings) and most of the time almost nothing was emitted. Additionally, it is not straightforward to obtain an accurate first guess from direct nuclear physics model, because even when an inventory of what has been generated in the core is hypothesized, it is difficult to know what has actually been released into the atmosphere and when. The related uncertainty of such an inventory would be barely known and in consequence difficult to use. That is the main reason why Nuclear Safety agencies (such as the Japanese Safety Agency or the French Institute of Radioprotection and Nuclear Safety (IRSN)) have provided first estimates constrained by the first observations (as from Chino et al. [2011], who used 17 observations to reconstruct a first estimate). But using these estimates as background terms in our inverse modeling process could be seen as an inversion crime and therefore should be avoided.

[66] However, to highlight the sensitivity of the retrieval to such an approach, we have performed a reconstruction using a nontrivial cesium-137 background term provided by the IRSN, focusing on the Japanese observation set.
The first guess and the retrieved source are plotted in Figure 13. The total released mass retrieved is greater than the one retrieved without the use of the background term $\left(1.9 \times 10^{16} \mathrm{~Bq}\right.$ instead of $\left.1.2 \times 10^{16} \mathrm{~Bq}\right)$. This is consistently explained by the fact that the analysis is strongly influenced by the first guess, specially in the early periods (mainly before 15 March and between 16 March and 19 March where it is impossible to distinguish between the first guess and the analysis profiles) where the observability was poor corresponding to a high uncertainty diagnosed in the retrieval with $\boldsymbol{\sigma}_{b}=\mathbf{0}$. This retrieved mass of $1.9 \times 10^{16} \mathrm{~Bq}$, which is not anymore meant to provide a lower bound but a full estimation, is remarkably consistent with the mass retrieved using the complete observation set $\left(1.9 \times 10^{16} \mathrm{~Bq}\right)$.

\subsubsection{Sensitivity to Physics Parametrization:}

\section{Example of the Dry Deposition}

[67] Model errors are expected to plague comparison between the model and distant observations. In particular a biased removal process should generate a strong discrepancy between the model and distant observations. That is why we have studied the sensitivity of the inverse modeling results to a change in the dry deposition velocity. We chose $0.15 \mathrm{~cm}$ $\mathrm{s}^{-1}$ for ${ }^{137} \mathrm{Cs}$ (instead of $0.2 \mathrm{~cm} \mathrm{~s}^{-1}$ ) and $0.3 \mathrm{~cm} \mathrm{~s}^{-1}$ for ${ }^{131} \mathrm{I}$ (instead of $0.5 \mathrm{~cm} \mathrm{~s}^{-1}$ ), which are the optimal parameter

Table 5. Summary of the Quantitative Results and Attached Uncertainty ${ }^{a}$

\begin{tabular}{lccc}
\hline \multicolumn{1}{c}{ Species } & $\begin{array}{c}\text { Released Activity }(\mathrm{Bq}), \\
\text { All Observations }\end{array}$ & $\begin{array}{c}\text { Released Activity (Bq), } \\
\text { Observations Over Japan }\end{array}$ & $\begin{array}{c}\text { Standard Deviation With } \\
\text { Perturbation of Observations }\end{array}$ \\
\hline Cesium-137 & $1.0 \times 10^{16}-1.9 \times 10^{16}$ & $\begin{array}{c}\text { Standard Deviation With Perturbation } \\
\text { of Observations and Background }\end{array}$ \\
Iodine-131 & $1.9 \times 10^{17}-7.0 \times 10^{17}$ & $\mathbf{1 . 9} \times \mathbf{1 0}^{\mathbf{1 7}}-\mathbf{3 . 8} \times \mathbf{1 0}^{\mathbf{1 6}}$ & $\begin{array}{c}15 \%-20 \% \\
5 \%-10 \%\end{array}$ \\
\hline
\end{tabular}

${ }^{\mathrm{a}}$ Values in bold are robust results. 


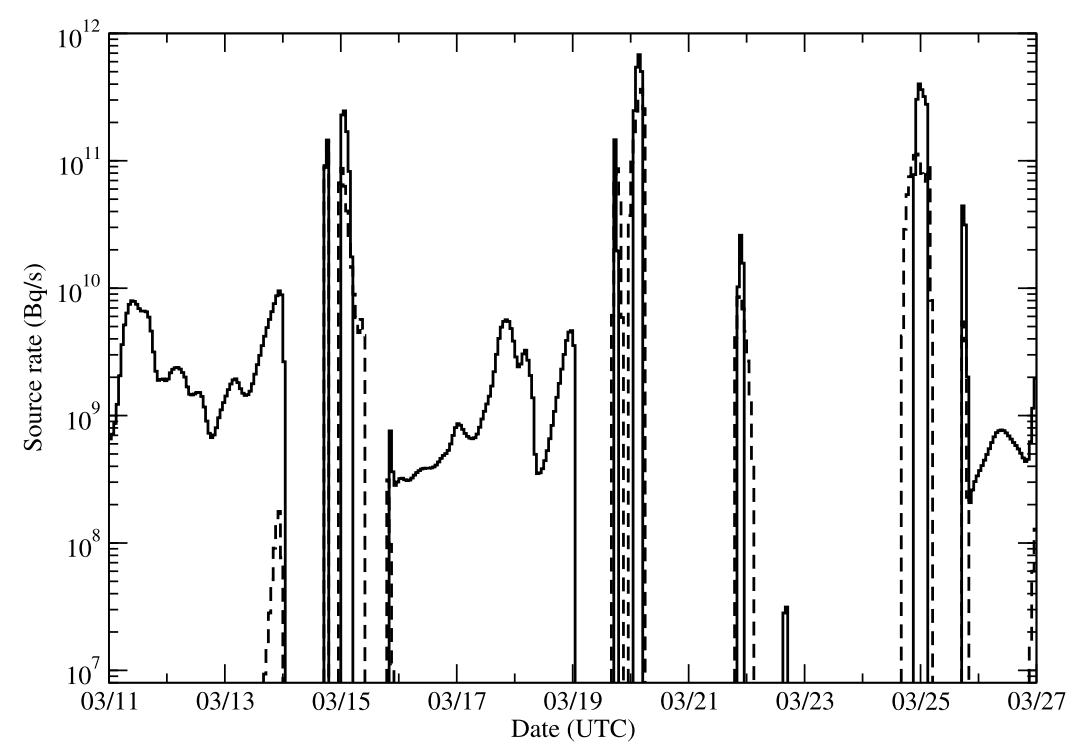

Figure 12. Reconstructed temporal profiles of cesium-137 under semi-Gaussian assumptions with the maximum likelihood method, using observations over Japan (dashed line) and all observations (full line).

values found by Bocquet [2012] for the Chernobyl accident dispersion. As expected, the total retrieved released activities are lower: the maximum likelihood estimates for ${ }^{137} \mathrm{Cs}$ are $0.9 \times 10^{16} \mathrm{~Bq}$ with the observations over Japan, and $1.6 \times 10^{16} \mathrm{~Bq}$ with all observations. For ${ }^{131} \mathrm{I}$, they are $1.6 \times 10^{17} \mathrm{~Bq}$ with the observations over Japan, and $1.9 \times 10^{17} \mathrm{~Bq}$ with all observations. If there is a gap reduction between retrieved activities for the two sets of observations and ${ }^{131} \mathrm{I}$, the gap is maintained for ${ }^{137} \mathrm{Cs}$.

\section{Conclusion}

[68] In this article, we have proposed efficient inverse modeling methods to reconstruct the Fukushima Daiichi source term of radionuclides available for long-range transport. We have assimilated publicly released air concentration activity measurements, which represent 267 observations for ${ }^{137} \mathrm{Cs}$ and 428 for ${ }^{131} \mathrm{I}$. Because of this small number of observations (compared to the 384 one hour time steps of the source terms) and the particular meteorological conditions leading to a poor observability of the release, the design of the priors is paramount. In particular, it is necessary to a posteriori estimate hyperparameters that characterize the prior errors.

[69] This analysis focused on two hyperparameters: $r$ the prior observation error standard deviation and $m$ the background error standard deviation. Three methods have been

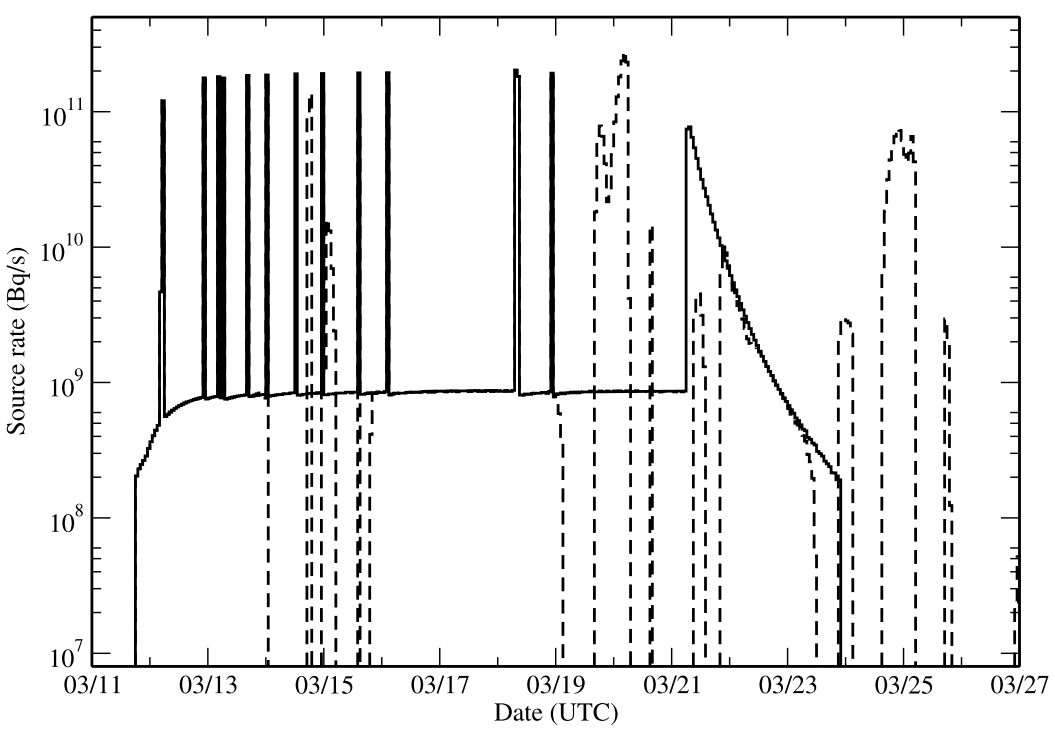

Figure 13. Reconstructed temporal profile of cesium-137 under semi-Gaussian assumptions with the maximum likelihood method, with the use of a nonnull first guess. The full line represents the IRSN first guess profile. The dashed line represents the retrieved source term. Note that the retrieved profile coincides with the first guess profile for the first unobserved sequence of peaks: 12-14 March. 
proposed to estimate these two parameters: (1) an L-curve method coupled with a $\chi^{2}$ diagnosis, (2) Desroziers's scheme, and finally (3) the maximization of the exact likelihood of the observation set as a function of $r$ and $m$. We have also assumed that the background error statistics are Gaussian or semi-Gaussian (i.e., forcing the reconstructed source term to be positive). In this latter case and using all the available observations, a lower bound of the total activity released in the atmosphere between 11 March and 26 March is estimated to be between $1.0 \times 10^{16} \mathrm{~Bq}$ and $1.9 \times 10^{16} \mathrm{~Bq}$ for ${ }^{137} \mathrm{Cs}$ and between $1.9 \times 10^{17} \mathrm{~Bq}$ and $7.0 \times 10^{17} \mathrm{~Bq}$ for ${ }^{131} \mathrm{I}$, which is comparable to the first estimates from the Nuclear Safety Commission of Japan (resp. $1.2 \times 10^{16} \mathrm{~Bq}$ and $1.5 \times 10^{17} \mathrm{~Bq}$ ) but represents probably an underestimation of the real emission. Using the most reliable methods in this case and the Japanese observations only, the lower bound estimate is $1.2 \times 10^{16} \mathrm{~Bq}$ for ${ }^{137} \mathrm{Cs}$, and in the range $1.9-3.8 \times 10^{17} \mathrm{~Bq}$ for ${ }^{131} \mathrm{I}$. They represent 5 to 10 times less emissions than the Chernobyl accident.

[70] The uncertainty of the reconstruction and its reduction have also been analyzed. Under Gaussian assumption, the posterior error covariance matrix has been used (mainly through its diagonal terms), whereas under semi-Gaussian assumptions, we used a Monte Carlo second-order sensitivity analysis to estimate the posterior error variance. In both cases, this analysis highlights the fact that some (between 2 and 3) time windows are well observed, during which the uncertainty is reduced, whereas some time windows are not well observed, with an uncertainty that remains high. These periods of high uncertainty on the reconstructed source correspond to configurations with a wind transporting the radioactive plume directly over the Pacific Ocean, the plume being eventually observed when arriving in North America, but with high uncertainties introduced by the dispersion model.

[71] In addition, a nonzero first guess inversion has been performed in the case of cesium-137 with the robust maximum likelihood method assimilating the Japanese observations. The total retrieved additivity was found to be consistently equivalent to the one of the inversion using all observations $\left(1.9 \times 10^{16} \mathrm{~Bq}\right)$. Indeed one can hope that some of the peaks of the nontrivial first guess unaccounted for by the Japanese set of observations will be explained by the additional observations.

[72] We have identified several ways to improve these reconstructions. First, we shall improve the accuracy of the dispersion model, for example the deposition parameterizations (dry deposition and/or wet scavenging). In such longdistance simulations, the uncertainties introduced by the numerical model may be very important. For instance, the deposition velocity over the ocean is understood to be significantly lower than over land, but its value remains unknown. Physical parameter inverse modeling techniques such as the one developed by Bocquet [2012] could be useful.

[73] It could also be interesting to implement inverse modeling methods that do not assume that observation error statistics are Gaussian (in addition to non-Gaussian background error statistics), such as in the work of Winiarek et al. [2011]. Finally in order to increase the number of available observation data, it can be interesting to develop methods which can take into account different types of observations, such as fallout data and gamma dose rates. The amount of available data would then increase substantially, but the prior uncertainties introduced in the system will also significantly increase (at least about ten different species, uncertainties on the deposition processes, precipitation, rainfall, etc.).

\section{Appendix A: Efficient Computation of the Semi-Gaussian Integral}

[74] In the semi-Gaussian case, the unapproximated value of the likelihood is given by equation (22):

$$
\begin{aligned}
p(\boldsymbol{\mu} \mid r, m)= & \frac{e^{-\frac{1}{2}\left(\boldsymbol{\mu}-\mathbf{H} \boldsymbol{\sigma}_{b}\right)^{\mathrm{T}}\left(\mathbf{R}+\mathbf{H B H} \mathbf{H}^{\mathrm{T}}\right)^{-1}\left(\boldsymbol{\mu}-\mathbf{H} \boldsymbol{\sigma}_{b}\right)}}{\sqrt{(2 \pi)^{d}\left|\mathbf{H B H} \mathbf{H}^{\mathrm{T}}+\mathbf{R}\right|}} \\
& \times 2^{N} \int_{\boldsymbol{\sigma} \geq 0} \frac{e^{-\frac{1}{2}\left(\boldsymbol{\sigma}-\boldsymbol{\sigma}_{a}\right)^{\mathrm{T}} \mathbf{P}_{a}^{-1}\left(\boldsymbol{\sigma}-\boldsymbol{\sigma}_{a}\right)}}{\sqrt{(2 \pi)^{N}\left|\mathbf{P}_{a}\right|}} \mathrm{d} \boldsymbol{\sigma},
\end{aligned}
$$

which can be seen as the product of the regular "Gaussian case" term (the fraction term) by a correction taking into account the positivity of the source term (the integral term). Estimating the value of the integral of a truncated multivariate normal distribution is a challenging task, studied in econometrics or numerical analysis. An extensive review of different methods has been performed by Hajivassiliou et al. [1996]. We chose to use the Geweke-Hajivassiliou-Keane (GHK) simulator, which is found by these authors to be the most reliable method. We have found that, in our context, is was more efficient than Gibbs sampling, which was for instance used by Lauvernet et al. [2009]. The principle of the GHK simulator is to draw samples following the truncated multivariate (N variables) Gaussian distribution $\mathcal{T} \mathcal{N}\left(\boldsymbol{\sigma}_{a}, \mathbf{P}_{a}, 0,+\infty\right)$.

[75] To draw a sample $\sigma_{*}$, the GHK simulator uses the Cholesky decomposition of the matrix $\mathbf{P}_{a}=\mathbf{L}^{\mathrm{T}} \mathbf{L}$, with $\mathbf{L}$ a lower triangular matrix. It reduces the problem to $\mathrm{N}$ draws of scalar random variables $\left(u_{i}\right)_{1 \leq i \leq N}$ following an univariate truncated normal distribution $\mathcal{\mathcal { T }} \mathcal{N}\left(0,1, \alpha_{i},+\infty\right)$ with the lower bounds $\alpha_{i}$ being given by

$$
\left\{\begin{array}{l}
\alpha_{1}=-\frac{\left[\boldsymbol{\sigma}_{a}\right]_{1}}{[\mathbf{L}]_{1,1}} \\
\alpha_{i}=-\frac{\left[\boldsymbol{\sigma}_{a}\right]_{i}}{[\mathbf{L}]_{i, i}}-\sum_{j=1}^{j=i-1} \frac{[\mathbf{L}]_{i, j}}{[\mathbf{L}]_{i, i}} u_{j} \quad \text { for } i=2, \ldots, N .
\end{array}\right.
$$

This method suggests that the draw of $\mathbf{u}=\left(u_{i}\right)_{1 \leq i \leq N}$ is made recursively. First, $\alpha_{1}$ is computed and $u_{1}$ is drawn from $\mathcal{T N}\left(0,1, \alpha_{1},+\infty\right)$, following the exponential accept-reject algorithm proposed by Robert [1995] if $\alpha_{1}>0.5$, a normal rejection algorithm if $\alpha_{1} \leq 0$, and a half-normal rejection algorithm otherwise. Then $\alpha_{2}\left(u_{1}\right)$ is computed and $u_{2}$ is drawn from $\mathcal{T} \mathcal{N}\left(0,1, \alpha_{2},+\infty\right)$ and so on. Then the desired sample $\sigma *$ is obtained via the transformation

$$
\sigma_{*}=\sigma_{a}+\mathbf{L u} .
$$

[76] In fact, this sampling scheme does not sample directly the target pdf, because of the dependence induced by the recursion. To correct the bias of the sample, one computes its importance sampling weight due to the difference 
between the proposal and the targeted distribution. The weight $w\left(\boldsymbol{\sigma}_{*}\right)$ is

$$
w\left(\boldsymbol{\sigma}_{*}\right)=\frac{1}{2^{N}} \prod_{i=1}^{i=N} \operatorname{erfc}\left(\frac{\alpha_{i}}{\sqrt{2}}\right) .
$$

Function erfc is the complementary error function defined by $\operatorname{erfc}(x)=\frac{2}{\sqrt{\pi}} \int_{x}^{+\infty} e^{-t^{2}} d t$. The value of the integral is then estimated with $n *$ samples using the empirical mean corrected by the importance sampling weights: $\frac{1}{n_{*}} \sum_{k=1}^{k=n *} w\left(\boldsymbol{\sigma}_{*}^{(k)}\right)$. In the GHK simulator, the $n_{*}$ samples are independent, so that the numerical computation of the integral can be massively parallelized. The value of the likelihood can then be easily screened for several values of $(r, m)$.

[77] Acknowledgments. This study has been supported by the IMMANENT project of Université Paris-Est and by the INSU/LEFEASSIM project ADOMOCA-2. We thank three reviewers for their helpful comments and suggestions. We gratefully acknowledge the help of Monika Krysta and Karine Sartelet with the data.

\section{References}

Abida, R., and M. Bocquet (2009), Targeting of observations for accidental atmospheric release monitoring, Atmos. Environ., 43, 6312-6327.

Anderson, J. L. (2007), An adaptive covariance inflation error correction algorithm for ensemble filters, Tellus, Ser. A, 59, 210-224.

Bocquet, M. (2005), Reconstruction of an atmospheric tracer source using the principle of maximum entropy. I: Theory, Q. J. R. Meteorol. Soc., 131, 2191-2208.

Bocquet, M. (2012), Parameter field estimation for atmospheric dispersion: Application to the Chernobyl accident using 4D-Var, Q. J. R. Meteorol. Soc., doi:10.1002/qj.961, in press.

Bocquet, M., C. A. Pires, and L. Wu (2010), Beyond Gaussian statistical modeling in geophysical data assimilation, Mon. Weather Rev., 138, 2997-3023.

Brandt, J., J. H. Christensen, and M. Frohn (2002), Modelling transport and deposition of caesium and iodine from Chernobyl accident using the DREAM model, Atmos. Chem. Phys., 2, 397-417.

Chapnik, B., G. Desroziers, F. Rabier, and O. Talagrand (2004), Properties and first application of an error-statistics tuning method in variational assimilation, Q. J. R. Meteorol. Soc., 130, 2253-2275.

Chapnik, B., G. Desroziers, F. Rabier, and O. Talagrand (2006), Diagnosis and tuning of observational error in a quasi-operational data assimilation setting, Q. J. R. Meteorol. Soc., 132, 543-565.

Chino, M., H. Nakayama, H. Nagai, H. Terada, G. Katata, and H. Yamazawa (2011), Preliminary estimation of release amounts of ${ }^{131} \mathrm{I}$ and ${ }^{137} \mathrm{Cs}$ accidentally discharged from the Fukushima Daiichi nuclear power plant into the atmosphere, J. Nucl. Sci. Technol., 48, 1129-1134.

Davoine, X., and M. Bocquet (2007), Inverse modelling-based reconstruction of the Chernobyl source term available for long-range transport, Atmos. Chem. Phys., 7, 1549-1564.

Dee, D. P. (1995), On-line estimation of error covariance parameters for atmospheric data assimilation, Mon. Weather Rev., 123, 1128-1145.

Delle Monache, L., et al. (2008), Bayesian inference and Markov chain Monte Carlo sampling to reconstruct a contaminant source on a continental scale, J. Appl. Meteorol. Climatol., 47, 2600-2613.

Desroziers, G., and S. Ivanov (2001), Diagnosis and adaptive tuning of observation-error parameters in a variational assimilation, $Q . J . R$. Meteorol. Soc., 127, 1433-1452.

Elbern, H., A. Strunk, H. Schmidt, and O. Talagrand (2007), Emission rate and chemical state estimation by 4-dimensional variational inversion, Atmos. Chem. Phys., 7, 3749-3769.

Enting, I. G. (2002), Inverse Problems in Atmospheric Constituent Transport, Cambridge Univ. Press, Cambridge, U. K.
Hajivassiliou, V., D. McFadden, and P. Ruud (1996), Simulation of multivariate normal rectangle probabilities and their derivatives: Theoretical and computational results, J. Econometrics, 72, 85-134.

Hansen, P. C. (1992), Analysis of discrete ill-posed problems by means of the L-curve, SIAM Rev., 34, 561-580.

Hansen, P. C. (2010), Discrete Inverse Problems: Insight and Algorithms, 225 pp., Soc. for Ind. and Appl. Math., Philadelphia, Pa.

Krysta, M., M. Bocquet, and J. Brandt (2008), Probing ETEX-II data set with inverse modelling, Atmos. Chem. Phys., 8, 3963-3971.

Lauvernet, C., J. M. Brankart, F. Castruccio, G. Broquet, P. Brasseur, and J. Verron (2009), A truncated Gaussian filter for data assimilation with inequality constraints: Application to the hydrostatic stability condition in ocean models, Ocean Modell., 27, 1-17.

Li, H., E. Kalnay, and T. Miyoshi (2009), Simultaneous estimation of covariance inflation and observation errors within an ensemble Kalman filter, Q. J. R. Meteorol. Soc., 135, 523-533.

Louis, J. (1979), A parametric model of vertical eddy fluxes in the atmosphere, Boundary Layer Meteorol., 17, 197-202.

Ménard, R., S. E. Cohn, L.-P. Chang, and P. M. Lyster (2000), Assimilation of stratospheric chemical tracer observations using a Kalman filter. Part I: Formulation, Mon. Weather Rev., 128, 2654-2671.

Michalak, A. M., L. Bruhwiler, and P. P. Tans (2004), A geostatistical approach to surface flux estimation of atmospheric trace gases, J. Geophys. Res., 109, D14109, doi:10.1029/2003JD004422.

Mitchell, H. L., and P. L. Houtekamer (1999), An adaptive ensemble Kalman filter, Mon. Weather Rev., 128, 416-433.

Pudykiewicz, J. (1989), Simulation of the Chernobyl dispersion with a 3-D hemispheric tracer model, Tellus, Ser. B, 41, 391-412.

Quélo, D., M. Krysta, M. Bocquet, O. Isnard, Y. Minier, and B. Sportisse (2007), Validation of the Polyphemus platform on the ETEX, Chernobyl and Algeciras cases, Atmos. Environ., 41, 5300-5315.

Robert, C. P. (1995), Simulation of truncated normal variables, Stat. Comput., $5,121-125$.

Saide, P., M. Bocquet, A. Osses, and L. Gallardo (2011), Constraining surface emissions of air pollutants using inverse modeling: Method intercomparison and a new two-step multiscale approach, Tellus, Ser. B, 63, 360-370.

Stohl, A., P. Seibert, G. Wotawa, D. Arnold, J. F. Burkhart, S. Eckhardt, C. Tapia, A. Vargas, and T. J. Yasunari (2011), Xenon-133 and caesium137 releases into the atmosphere from the Fukushima Dai-ichi nuclear power plant: Determination of the source term, atmospheric dispersion, and deposition, Atmos. Chem. Phys. Discuss., 11, 28,319-28,394.

Villani, M. G., P. Bergamaschi, M. Krol, J. F. Meirink, and F. Dentener (2010), Inverse modeling of European $\mathrm{CH}_{4}$ emissions: Sensitivity to the observational network, Atmos. Chem. Phys., 10, 1249-1267.

Vogel, C. R. (2002), Computational Methods for Inverse Problems, Frontiers Appl. Math., vol. 23, 183 pp., Soc. for Ind. and Appl. Math., Philadelphia, Pa.

Wahba, G. (1990), Spline Models for Observational Data, CBMS-NSF Reg. Conf. Ser. Appl. Math., vol. 59, 180 pp., Soc. for Ind. and Appl. Math., Philadelphia, Pa.

Winiarek, V., J. Vira, M. Bocquet, M. Sofiev, and O. Saunier (2011), Towards the operational estimation of a radiological plume using data assimilation after a radiological accidental atmospheric release, Atmos. Env., 45, 2944-2955.

Wu, L., M. Bocquet, T. Lauvaux, F. Chevallier, P. Rayner, and K. Davis (2011), Optimal representation of source-sink fluxes for mesoscale carbon dioxide inversion with synthetic data, J. Geophys. Res., 116, D21304, doi:10.1029/2011JD016198.

Yee, E., F. S. Lien, A. Keats, and R. D'Amours (2008), Bayesian inversion of concentration data: Source reconstruction in the adjoint representation of atmospheric diffusion. J. Wind Eng. Ind. Aerodyn., 96, 1805-1816.

M. Bocquet and V. Winiarek, CEREA, École des Ponts ParisTech and EDF R\&D, 6-8 Ave. Blaise Pascal, Cité Descartes, Champs-sur-Marne, F-77455 Marne la Vallée CEDEX 2, France. (marc.bocquet@cerea.enpc.fr; victor.winiarek@cerea.enpc.fr)

A. Mathieu and O. Saunier, Institute of Radiation Protection and Nuclear Safety, BP 17, F-92262 Fontenay-aux-Roses, France. 